\title{
Recurrent ubiquitin B silencing in gynecological cancers establishes dependence on ubiquitin C
}

\author{
Alexia T. Kedves, ${ }^{1}$ Scott Gleim, ${ }^{1}$ Xiaoyou Liang, ${ }^{1}$ Dennis M. Bonal, ${ }^{2}$ Frederic Sigoillot, ${ }^{1}$ Fred Harbinski, ${ }^{1}$ Sneha Sanghavi, ${ }^{3}$ \\ Christina Benander, ${ }^{1}$ Elizabeth George, ${ }^{1}$ Prafulla C. Gokhale, ${ }^{2}$ Quang-De Nguyen, ${ }^{2}$ Paul T. Kirschmeier, ${ }^{2}$ Robert J. Distel, ${ }^{2}$ \\ Jeremy Jenkins, ${ }^{1}$ Michael S. Goldberg, ${ }^{2}$ and William C. Forrester ${ }^{1}$
}

${ }^{1}$ Chemical Biology and Therapeutics, Novartis Institutes for Biomedical Research, Cambridge, Massachusetts, USA. ²Dana-Farber Cancer Institute, Boston, Massachusetts, USA.

${ }^{3}$ Neurosciences, Novartis Institutes for Biomedical Research, Cambridge, Massachusetts, USA.

\begin{abstract}
Transcriptional repression of ubiquitin $B(U B B)$ is a cancer-subtype-specific alteration that occurs in a substantial population of patients with cancers of the female reproductive tract. UBB is 1 of 2 genes encoding for ubiquitin as a polyprotein consisting of multiple copies of ubiquitin monomers. Silencing of UBB reduces cellular UBB levels and results in an exquisite dependence on ubiquitin $C(U B C)$, the second polyubiquitin gene. UBB is repressed in approximately $30 \%$ of high-grade serous ovarian cancer (HCSOC) patients and is a recurrent lesion in uterine carcinosarcoma and endometrial carcinoma. We identified ovarian tumor cell lines that retain UBB in a repressed state, used these cell lines to establish orthotopic ovarian tumors, and found that inducible expression of a UBC-targeting shRNA led to tumor regression, and substantial long-term survival benefit. Thus, we describe a recurrent cancer-specific lesion at the level of ubiquitin production. Moreover, these observations reveal the prognostic value of UBB repression and establish UBC as a promising therapeutic target for ovarian cancer patients with recurrent $U B B$ silencing.
\end{abstract}

\section{Introduction}

Target discovery in oncology has focused predominantly on 2 basic principles: oncogene addiction (1) and synthetic lethality (2). Identifying cancer-specific gain-of-function alterations in oncogenes such as $A B L, B R A F, E G F R$, and PI3K has fueled the development of potent and selective kinase inhibitors that antagonize the addiction of cancer cells to a defined oncogenic driver or pathway. By contrast, loss-offunction mutations, most notably in tumor suppressor genes, are not readily druggable, motivating the search for synthetic lethal targets (3).

These approaches are complemented by large-scale bioinformatic analysis of tumor profiling data. Coordinated efforts such as The Cancer Genome Atlas (TCGA) project seek to catalog several measurable features of tumor cells, including copy number alterations, mutations, expression, and epigenetic states, and have enabled the classification of patients into specific disease subtypes as well as the identification of new, actionable targets. Glioblastoma, for example, while still diagnosed based on anatomy and histopathology (4), has been further separated into subtypes based on genetic alterations, expression signatures, and long-term survival $(5,6)$. This approach identified a subset of patients with gain-offunction mutations in a gene that had not previously been linked to cancer, IDH1 (6), leading to the development of inhibitors that are being investigated in clinical trials (7).

Related Commentary: p. 4228

Conflict of interest: The authors have declared that no conflict of interest exists. Submitted: January 17, 2017; Accepted: October 9, 2017. Reference information: J Clin Invest. 2017;127(12):4554-4568. https://doi.org/10.1172/JCI92914.
Such new approaches are imperative because there are many cancer patients for whom treatment options have not substantively changed. The mainstay treatment for women with high-grade serous ovarian cancer (HGSOC) remains cisplatin, which was introduced in the late 1970s (8), and while survival rates have largely unchanged since then (9), emerging therapeutic strategies that include surgical improvements, use of anti-VEGF antibodies, and recent approvals for PARP inhibitors indicate that significant progress is being made (10-12). While profiling in HGSOC has led to important insights for the molecular classification of ovarian cancer subtypes, there persists a gap at the level of finding lesions that act as oncogenic drivers and are validated as clinically relevant targets in these patients (13-15). Synthetic lethality, commonly based on tumor suppressor loss, emerges as an alternative approach. Indeed, PARP inhibitors were recently approved for the treatment of a subset of ovarian cancer patients who harbor $B R C A$ mutations $(16,17)$.

In the context of this major unmet medical need, we continued to look for stratification-based opportunities that could serve as the starting points for a next generation of treatment options using the TCGA data set. This approach led to the identification of a recurrent alteration in the expression of the ubiquitin gene $U B B$, which is silenced in a significant subset of patients with HGSOC as well as in other tumors of the gynecological tract including uterine carcinosarcoma (UCS) and uterine corpus endometrial carcinoma (UCEC). Several cell lines profiled as part of the Cancer Cell Line Encyclopedia (CCLE) also contain UBB in a transcriptionally repressed state, enabling us to assess the therapeutic value of this observation.

Levels of cellular ubiquitin are determined by the expression of 4 genes in the human genome that encode for ubiquitin protein that is identical at the amino acid level. The 4 genes are 
A

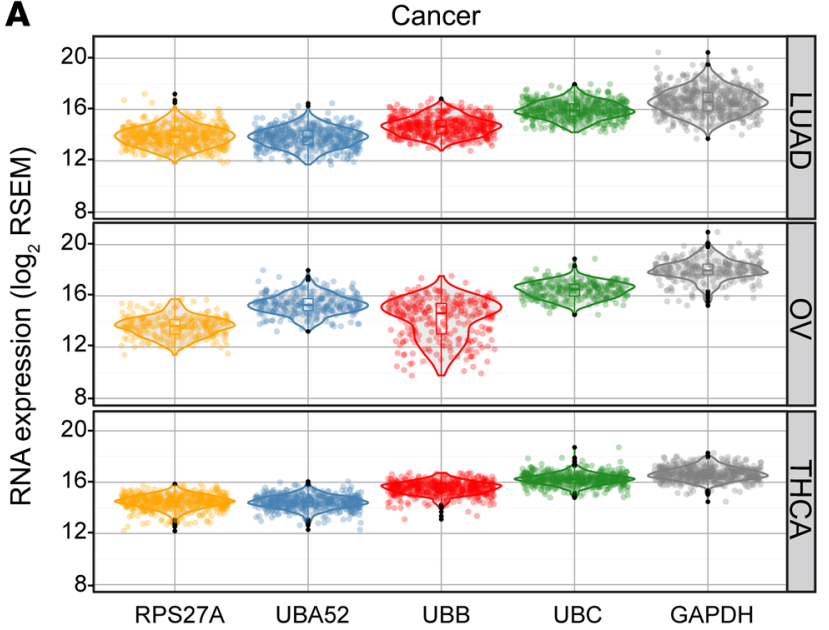

B

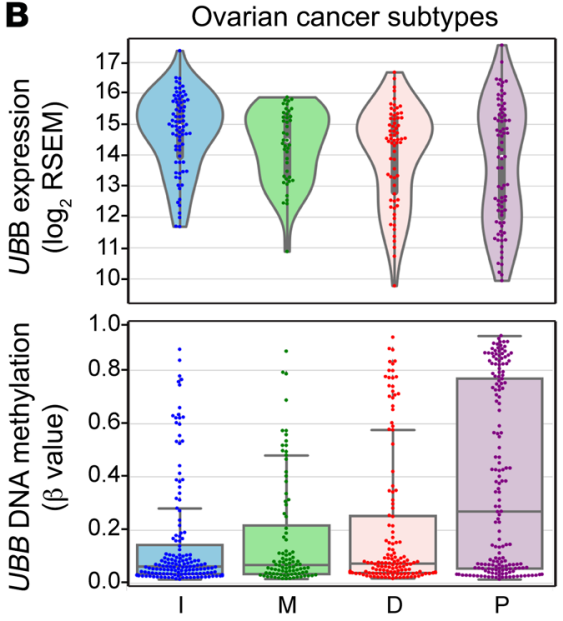

C

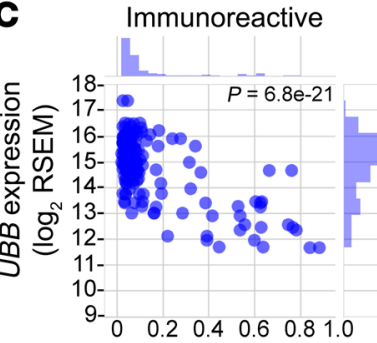

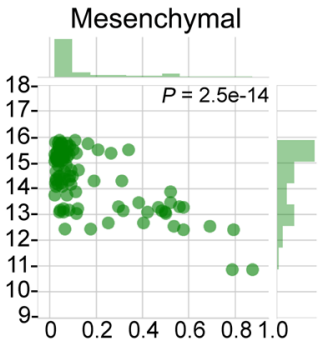

UBB DNA methylation ( $\beta$ value)

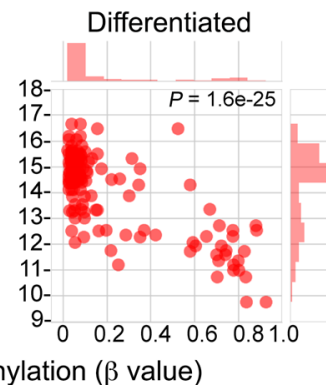

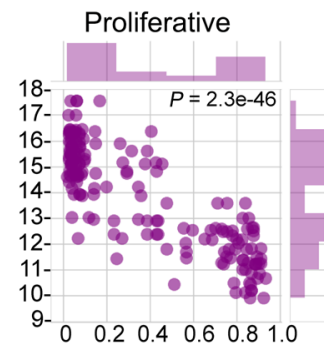

Figure 1. TCGA analysis identifies UBB silencing in HCSOC. (A) Expression of the 4 human genes that encode for ubiquitin - RPS27A (yellow), UBA52 (blue), UBB (red), and UBC (green) - along with GAPDH (gray), in 3 TCGA tumor types - lung (LUAD), ovarian (OV), and thyroid (THCA). RNASeq data are displayed as gene-level $\log _{2}$ RSEM values. Each data point represents a patient-specific RNA level for the indicated gene, with violin plot profiles indicating the derived probability density of each population. (B) UBB expression and DNA methylation in subtypes previously established by TCGA is shown in the top and bottom panels, respectively. Subtypes defined based on transcriptional signatures are immunoreactive (I), mesechymal (M), differentiated (D), and proliferative (P). Methylation levels are reported as $\beta$ values from 0 (no methylation) to 1.0 (full methylation). (C) Correlation plots and corresponding $P$ values show subtype-specific methylation and expression of $U B B$ (associated Pearson $R^{2}$ and $P$ values: immunoreactive $=-0.66,6.8 \mathrm{e}^{-21}$; mesenchymal $=$ $-0.69,2.5 \mathrm{e}^{-14}$; differentiated $=-0.74,1.6 \mathrm{e}^{-25}$; proliferative $\left.=-0.84,2.3 \mathrm{e}^{-46}\right)$. Colors by transcriptional subtype correspond to $\mathbf{B}$.

RPS27A, UBA52, UBB, and UBC. In each of these loci, ubiquitin is encoded as a polyprotein that is cleaved into the canonical 76-residue ubiquitin protein. RPS27A and UBA52 encode a fusion of ubiquitin and a small and large ribosomal protein, respectively, while mRNA from the $U B B$ and $U B C$ loci contain the 228 nucleotides encoding the 76 -amino acid ubiquitin protein as a multiple of 3 and 9 head-to-tail repeats, respectively (18). In all cases, the 76-amino acid ubiquitin protein is fully conserved and, in this way, contributes to the common cellular pool of ubiquitin that is, in turn, deployed by ubiquitin E3 ligases that append ubiquitin moieties onto proteins to regulate their stability, localization, and activity (19).

Ubiquitin genes serve housekeeping functions and levels of free ubiquitin must be maintained to sustain homeostasis. Finding a cancer-specific context in which one of these genes is recurrently silenced establishes a rationale for exploring the impact of this event for tumorigenesis and the therapeutic opportunities made possible as a result of this lesion. We have explored the clinical value of $U B B$ silencing and find that knockdown of UBC in these cells leads to the depletion of cellular ubiquitin pools below a viability threshold and show that this effect is specific and rescuable. We confirm the relevance of these observations by finding $U B B$ silencing in a subset of gynecological tumors and the ability to exploit this lesion to induce remissions in an in vivo xenograft study.

Pan-cancer TCGA data analysis has led to the potentially novel finding that $U B B$ silencing, while infrequent in most cancer types, occurs in approximately $15 \%$ to $30 \%$ of UCS, UCEC, and HGSOC and therefore is enriched in a subset of gynecological cancers. Our functional studies provide a basis for considering $U B C$ as essential to the survival of cells lacking $U B B$ expression both in vitro and in vivo. We propose that the combined loss of ubiquitin from both $U B B$ and $U B C$ leads to a catastrophic deficiency of ubiquitin. Synthetic lethality in these cells therefore exploits a tumor-cell-specific reduction in ubiquitin that is sensitive to further ubiquitin depletion and validates $U B B$ silencing as a therapeutic opportunity.

\section{Results}

UBB silencing in human gynecological cancer. We examined expression data from TCGA samples across 27 tumor types (www. cancergenome.nih.gov) and found that the polyubiquitin gene $U B B$ is decreased in a subset of patients from the ovarian (OV) data set consisting of samples from patients with HGSOC (ref. 20 
A

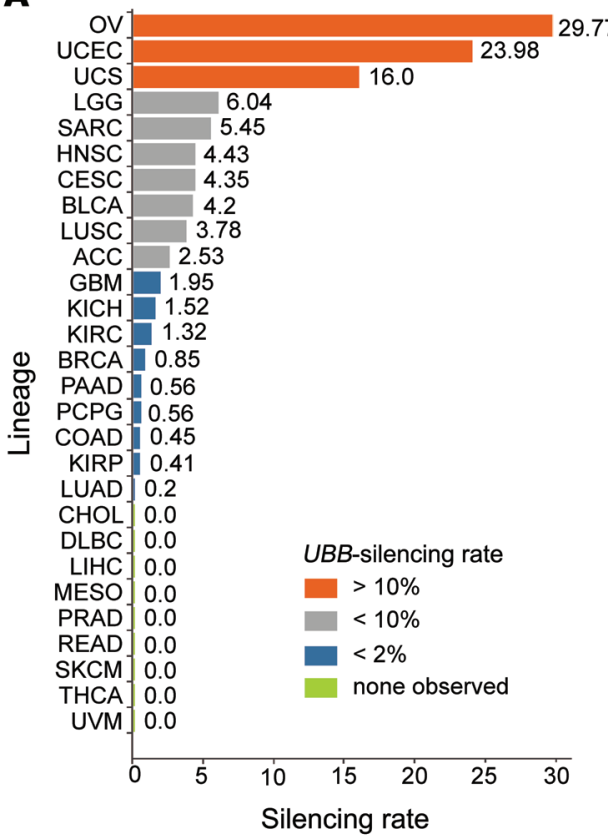

B
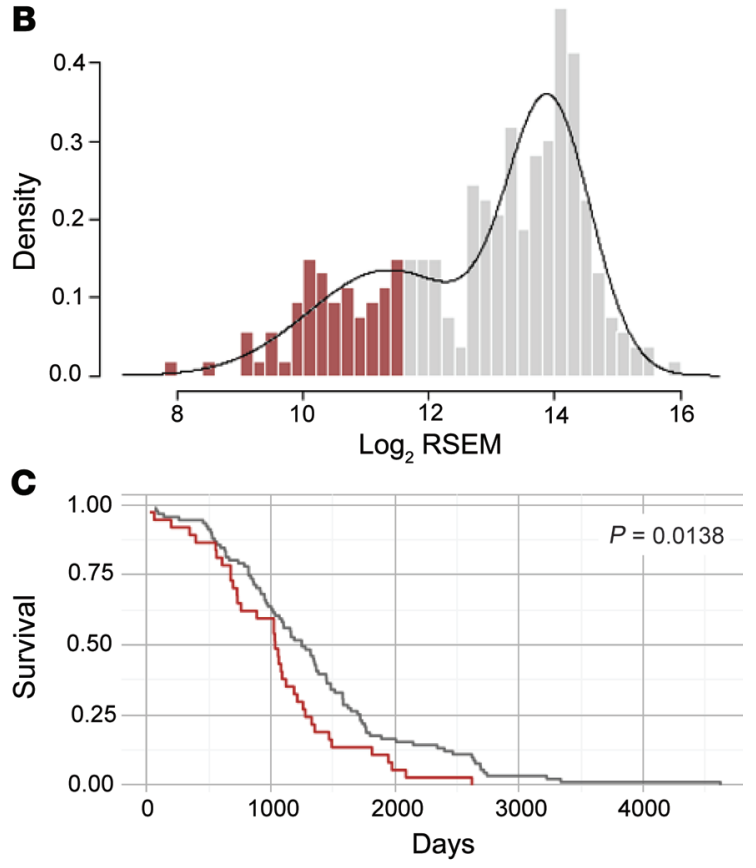

Figure 2. UBB silencing in gynecological cancers is associated with poor outcomes in HCSOC. (A) The frequency of decreased $U B B$ expression is shown for each cancer type. The ratio of $U B C / U B B$ mRNA, on a per-patient basis, was determined and the percentage of patients having a $U B C / U B B$ ratio greater than 1.25 is referred to as the silencing rate. A color scheme is used to highlight tumor types with comparable rates of $U B B$ silencing. (B) Analysis of $U B B$ in HCSOC indicates a bimodal distribution of $\log _{2}(U B B)$ expression levels based on a Caussian mixture model, illustrated by kernel density estimates. Dark red color indicates samples within the lowest quartile of expression (dark red $=\log _{2}[U B B] \leq Q_{1}$, gray $=\log { }_{2}[U B B]>Q_{1}$ ). (C) Kaplan-Meier survival curves of HCSOC patient survival stratified by the lowest quartile of UBB expression (dark red) demonstrates significantly poorer outcomes than the patients with higher UBB expression tumors (gray) $(P=0.0138)$.

and Figure 1A). The RNA sequencing (RNASeq) data are shown for each of the 4 ubiquitin-encoding genes along with GAPDH as an internal control for sample quality and distribution normalcy. We find that all ubiquitin-encoding genes, RPS27A, UBA52, $U B B$, and $U B C$ are expressed in tight, unimodal distributions, with the exception of $U B B$ in HGSOC (labeled OV in Figure 1). In this tumor type, $U B B$ expression shows an atypical and broad distribution and indicates that in many patients $U B B$ expression is subject to transcriptional downregulation.

To understand the relevance of this specific silencing event in the context of previously defined disease subtypes within the HGSOC data set $(20,21), U B B$ expression was examined in each of the 4 established subtypes: immunoreactive, mesenchymal, differentiated, and proliferative (Figure 1B). Our results indicate that low $U B B$ expression based on RNASeq by expectation-maximization $(\mathrm{RSEM})$ values $\left(\log _{2} \mathrm{RSEM}\right.$ values $\left.\leq 12\right)$ occurs preferentially in the proliferative and differentiated subtypes. In these subtypes, the distribution of $U B B$ expression is bimodal, with more tumors in the proliferative subtype expressing low levels of $U B B$.

To gain additional insight into the possible basis for downregulation of $U B B$ expression, we assessed TCGA patient-specific DNA methylation data from primary tumors, revealing a strong enrichment for $U B B$ methylation in the proliferative subtype (Figure $1 \mathrm{~B}$ ). In these plots, the degree of methylation is the $\beta$ value (see legend) and reflects an Illumina sequencing approach that quantifies the conversion of unmethylated $\mathrm{CpG}$ into TpG following bisulfite treatment (22). The $\beta$ value reflects the ratio of methylation at a specific locus and ranges from 0 (no methylation) to 1.0 (full methylation). These results show that $U B B$ is strongly methylated in the proliferative subtype, with the mean methylation higher in this subtype than the top quartile in the differentiated subtype, the group with the next highest level of methylation. There is also a substantial outlier population of heavily methylated $U B B$ alleles ( $\beta$ values $>0.8$ ) in the proliferative subtype.

These data can be combined to consider the correlation between expression and DNA methylation within each subtype, on a per-patient basis (Figure $1 C$ ). The $P$ values show that for all subtypes $U B B$ expression is highly significantly correlated with its methylation status. In other words, $U B B$ expression occurs when the gene is unmethylated and is silenced when the gene is heavily methylated. Together, these results provide compelling evidence that $U B B$ silencing and DNA methylation occurs preferentially in the proliferative subtype of ovarian cancer.

The human polyubiquitin genes $U B B$ and $U B C$ are typically expressed at roughly equivalent levels in cell lines, normal tissues, and tumor samples. To consider $U B B$ expression more broadly across TCGA samples, we used the ratio of $U B C$ to UBB mRNA to identify relative expression outliers. This approach provides an internally controlled, patient-centric normalization for sample variability. We use a $U B C / U B B$ ratio cutoff of greater than 1.25 as a measure of $U B B$ silencing rate. This value is defined by the upper inner fence, a standard statistical measure of outlier status defined as $Q 3+(1.5 \times I Q)$ (see Methods), and taking this approach were able to establish a rate of $U B B$ silencing for each tumor type 
Table 1. The Mantel-Haenszel log-rank test on the Kaplan-Meier estimate of survival

$\begin{array}{lcccc}\text { RSEM } & \text { Observed (0) } & \text { Expected (E) } & (\mathbf{O}-\mathrm{E})^{2} / \mathrm{E} & (\mathbf{0}-\mathrm{E})^{2} / \mathrm{V} \\ U B B>01 & 91 & 102 & 1.18 & 6.06 \\ U B B \leq 01 & 37 & 26 & 4.63 & 6.06\end{array}$

The Mantel-Haenszel log-rank test was used to evaluate the difference between the Kaplan-Meier survival curves for TCGA HGSOC patients stratified according to UBB expression level ( $\log _{2}$ RSEM). The UBB-low group $(U B B \leq Q 1)$ was defined by $U B B$ expression levels in the lowest quartile, and demonstrated a significant survival difference compared with other patients $\left(\chi^{2}=6.06, P=0.0138\right)$. The significance threshold was set at the $95 \%$ confidence interval $(P$ value $<0.05)$. O, observed deaths; $\mathrm{E}$, expected deaths; $\mathrm{V}$, variance.

(Figure 2A). This analysis reveals that $U B B$ silencing $(U B C / U B B$ ratio greater than 1.25) is found in many different types of cancer but is most prevalent in 3 cancers of the female reproductive tract: 16\% UCS, 24\% UCEC, and 29\% HGSOC (OV). These 3 cancer types define a distinct group (orange bars) where $U B B$ silencing occurs at a relatively high frequency. The second group of tumors (gray bars) silences $U B B$ at a much lower rate, between $2.5 \%$ and $6 \%$, and this is followed by a gradual tapering off in the rate of $U B B$ silencing in other tumor types. Low $U B B$ expression outliers across the TCGA data can be seen on a patient-by-patient basis in many tumor types but is clearly not equally distributed (Supplemental Figure 1A; supplemental material available online with this article; https://doi.org/10.1172/JCI92914DS1). The decrease in $U B B$ expression is not seen in a similar analysis in normal tissues (Supplemental Figure 1B), suggesting that $U B B$ repression in tumors is guided by tissue-specific mechanisms and occurs at a high frequency in a subset of gynecological cancers.

To understand the impact that $U B B$ silencing may have on clinical outcomes, we considered the distribution of $U B B$ expression among HGSOC in the TCGA data set. Expression is best described as a bimodal distribution, based on a Gaussian mixture model, with low and normal $U B B$ expression groups (colored red and gray, respectively, Figure 2B). Stratification of long-term survival data into 2 groups based on $U B B$ expression levels in tumor samples $\left(\log _{2}[U B B] \leq Q 1\right.$ or $\left.\log _{2}[U B B]>Q 1\right)$ demonstrated the lowest quartile of $U B B$ expression to correlate with poor patient outcome in overall survival compared with the remainder $(P$ value $=0.0138)($ Figure $2 \mathrm{C})$. The Mantel-Haenszel log-rank test was used to evaluate the significance between Kaplan-Meier survival curves (Table 1).

Silencing of $U B B$ establishes dependence on $U B C$. As a basis for considering the functional implications of these observations, we wanted to know if we could identify cell lines expressing $U B B$ at low levels, presumably derived from patients such as those described above. We surveyed expression of ubiquitin-encoding genes across the CCLE, which consists of more than 1,000 cell lines from 24 tumor types (ref. 23 and Figure $3 \mathrm{~A}$ ). We find a subset of cell lines in which $U B B$ expression is strongly decreased, while expression of the 3 other ubiquitin genes in these cells remains within a relatively tight distribution shared among all cells. This is also seen in box plots representing the same data (Figure 3B), which clearly reveal a group of outliers with low levels of $U B B$ expression. Similar to the TCGA data, we also find that $U B B$ silencing in these cells is correlated with increased DNA methylation (W. Forrester, unpublished observations). Importantly, we have identified a number of cell lines in which $U B B$ appears to be transcriptionally repressed and, if confirmed, will enable us to pursue functional studies to determine the translational value of this finding.

This analysis identified 26 cell lines in which $U B B$ expression is strongly suppressed (Supplemental Table 1). These data indicate that $U B B$ silencing is regulated independently of other ubiquitin genes and is likely to be maintained in cell lines derived from tumors in which $U B B$ has been turned off. The highest frequency of $U B B$ repression occurs in cell lines derived from ovarian and endometrial cancers, and almost $15 \%$ of cell lines derived from these lineages express very low levels of $U B B$ (Supplemental Table 2). This representation parallels our observations of $U B B$ expression in the TCGA data set and provides independent support that repression of $U B B$ is linked to gynecological cancers.

To study this in more detail, we selected a group of cell lines from different tumor types based on $U B B$ expression levels. We sought to confirm $U B B$ expression in these cell lines and, after obtaining and expanding these cells, performed RNA analysis using gene-specific probes that distinguish between each of the 4 ubiquitin-encoding genes. Results from this study demonstrate that our measurements of $U B B$ expression closely reproduce results from data collected by a high-throughput profiling platform (Figure 3C). Importantly, we observe potent $U B B$ mRNA reductions in a subset of cancer cell lines, hereafter referred to as $U B B^{\mathrm{LO}}$ lines including CAL33 (tongue squamous cell carcinoma), HMCB (melanoma), JHOM1 (ovarian mucinous adenocarcinoma), KS-1 (glioblastoma), KYSE150 (esophageal squamous cell carcinoma), OVK18 (ovarian endometrioid adenocarcinoma), and OVCAR8 (HGSOC).

By virtue of the number of ubiquitin repeats, the polyubiquitin genes $U B B$ and $U B C$ can contribute disproportionately to the pool of cellular ubiquitin protein on a per-transcript basis, relative to the UBA52 and RPS27A genes which each encode a single ubiquitin polypeptide. We wanted to consider whether cells repressed for $U B B$ had an increased dependence on $U B C$ to maintain their ubiquitin pool. To address this, we silenced $U B C$ in cell lines with normal or reduced levels of $U B B$. siRNA-mediated knockdown of the essential gene PLK1 was used as a transfection efficiency control and induces a strong viability phenotype in transfected cells as measured by CellTiter-Glo. All cell lines in this panel, including both $U B B^{\mathrm{LO}}$ (red) and $U B B^{\mathrm{WT}}$ (black) lines, are dependent on PLK1. By contrast, knockdown of $U B C$ produces a viability phenotype only in the $U B B^{\mathrm{LO}}$ cells (Figure 3D).

To assess cellular outcomes resulting from $U B C$ knockdown, we followed cells in a time course after siRNA transfection to better understand if cells were undergoing a cell cycle arrest or a cell death response. The cellular response was observed using real-time microscopy with the Incucyte apparatus that allowed us to look at cell morphology and, in combination with a fluorogenic caspase substrate dye (24), allowed us to additionally monitor the induction of caspases involved in executing the apoptotic program (Figure 4). $U B B^{\mathrm{LO}}$ (OVCAR8) and $U B B^{\mathrm{WT}}$ (OC316) cells transfected with siRNA targeting PLK1 show a morphology, previ- 
A

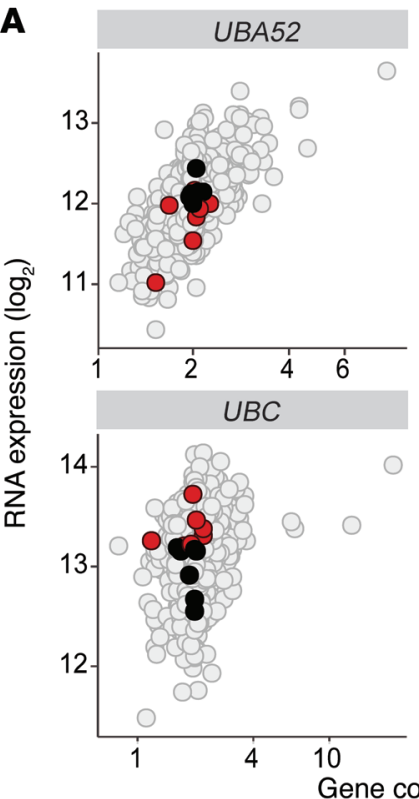

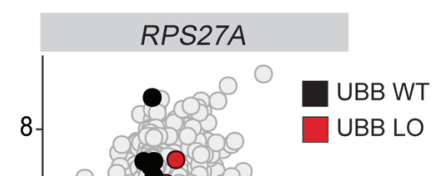

0 .

6 .

6.

1
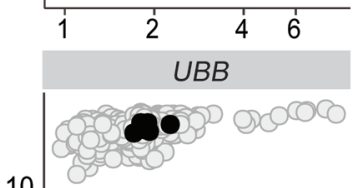

B
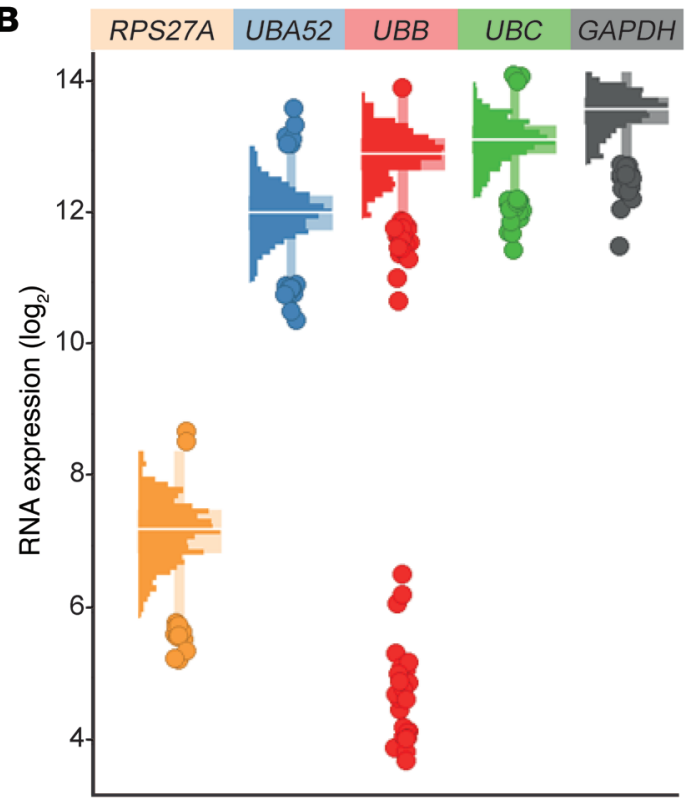

C

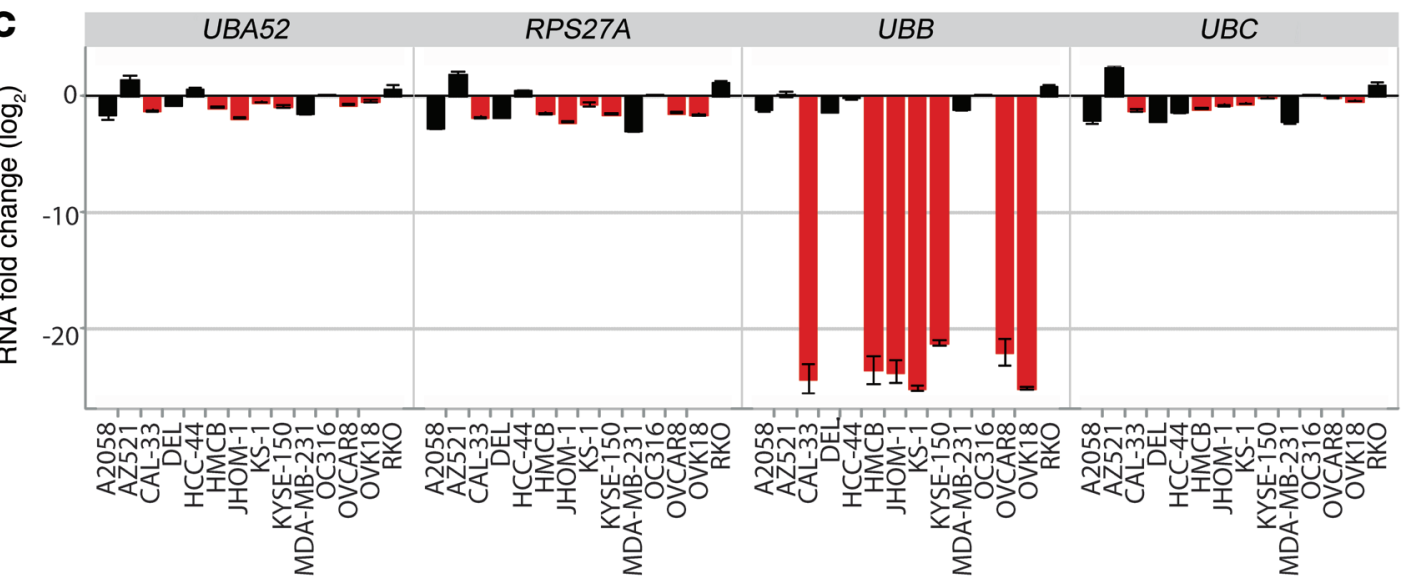

D

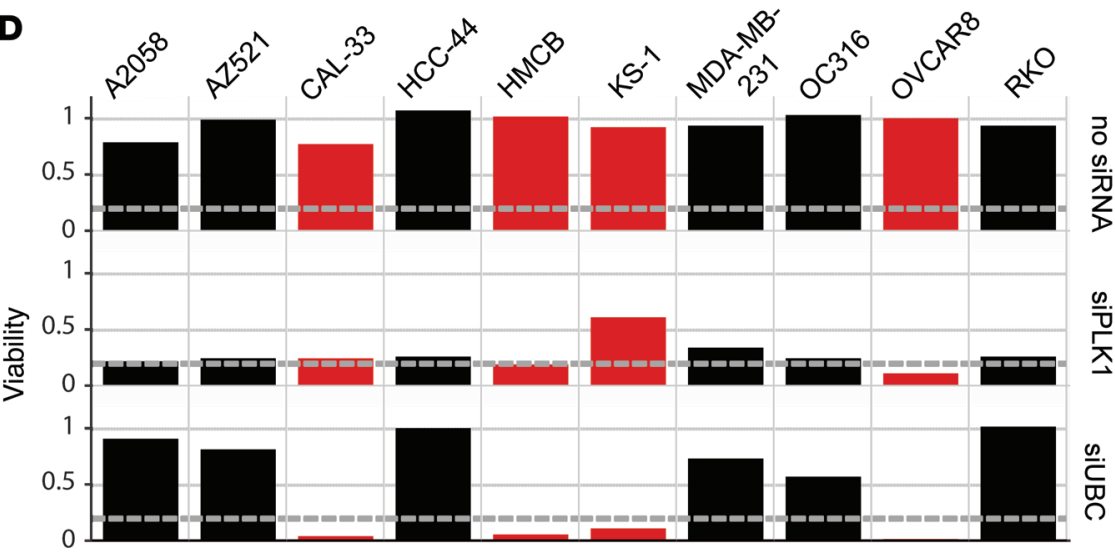

Figure 3. Ubiquitin expression and synthetic lethality. (A) Gene expression versus gene copy number is shown for each of the 4 ubiquitin-encoding genes across all 1,053 cell lines from the CCLE. Each dot represents a single cell line. Note that the range of $y$-axis values reflects an expression range specific for each gene. Cell lines highlighted in red and black were selected for subsequent studies to validate levels of ubiquitin expression. The $x$ axis shows the number of gene copies per diploid genome. (B) Box plots of data from A directly comparing expression of ubiquitin genes. Expression is a unimodal distribution for all ubiquitin genes except for UBB, which exhibits a substantial outlier population. GAPDH is included as a control. (C) Selected cell lines

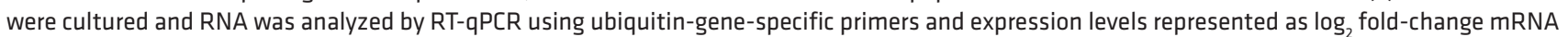
relative to OC316. Data bars are colored red or black corresponding to the scheme in $\mathbf{A}$. Biological quadruplicates with mean \pm SEM are shown. This study was repeated twice. (D) UBB ${ }^{10}$ cell lines (red) are sensitive to UBC knockdown. Cell lines were transfected with 5 nM siRNAs targeting PLK1 or UBC. Mock transfections performed without siRNA are shown and results from each cell line are normalized to 1.0 based on viability of cells treated with nontargeting siRNA (data not included). Dashed lines indicate 10\% viability level. CellTiter-Glo was added 72 hours after transfection. Results shown were singlicates and this experiment was performed 3 times independently. 

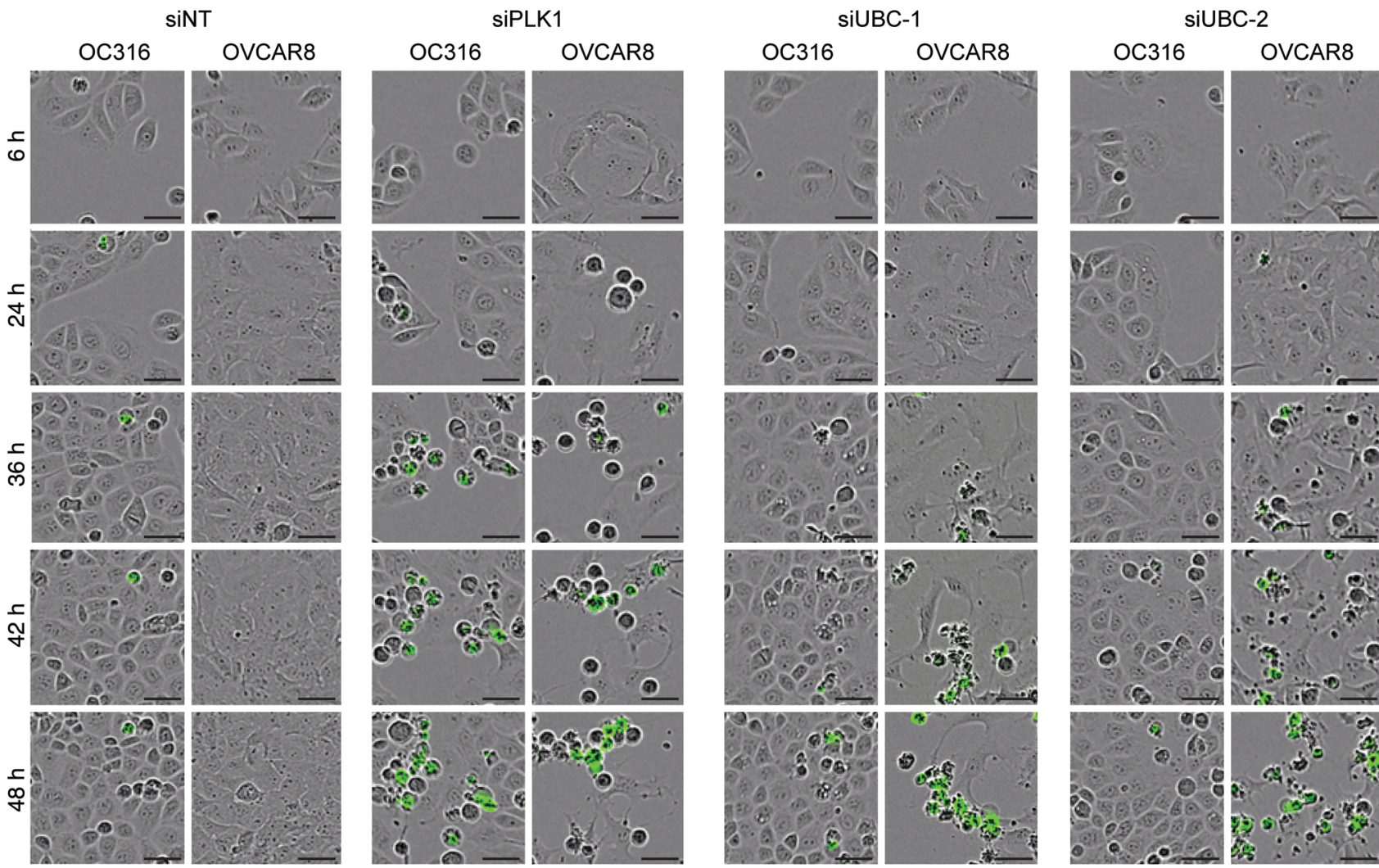

Figure 4. Time-dependent cell fate outcomes following UBC knockdown. Cells were transfected with siRNAs for UBC and PLK1 and were imaged continuously in the presence of a fluorogenic caspase substrate. Representative images show cell morphology and induction of caspase-3/7 activity over a period of 2 days. Caspase activation results in cleavage of a dye precursor resulting in fluorescence and is seen in both UBB ${ }^{\mathrm{LO}}$ (OVCAR8) and UBB ${ }^{\mathrm{WT}}$ (OC316) cells after knockdown of $P L K 1$. Fluorescence is seen following knockdown of $U B C$ only in $U B B^{\llcorner 0}$ cells. Scale bars: $50 \mu \mathrm{m}$. Original magnification, $x 10$. This study was done twice.

ous described, that is characteristic of cells unable to exit $\mathrm{G} 2$ phase (25) and this can be seen starting at 24 hours. Fluorescent signal resulting from caspase-3/7 activation can be seen starting at 36 hours and increases over time. Transfection with siRNAs against $U B C$ produces fluorescence exclusively in the $U B B^{L O}$ cells starting at 42 hours. The bright-field images also show disruption to the structural integrity of the $U B B^{\mathrm{LO}}$ cells starting at 36 hours. The $U B B^{\text {WT }}$ (OC316) cells transfected with UBC siRNAs appear unaffected, similar to the impact of transfecting these cells with the nontargeting control siRNA (siNT). These observations indicate that $U B C$ knockdown in $U B B^{\mathrm{LO}}$ cells results in cell death, consistent with the strong viability effects seen above.

To consider this phenotype in more detail, a panel of lines consisting of $4 U B B^{\mathrm{LO}}$ (red) and $6 U B B^{\mathrm{WT}}$ lines (black) from different lineages were transfected with a dose titration of validated, gene-specific siRNAs while maintaining total siRNA concentration at $20 \mathrm{nM}$ using siNT as filler (Figure 5A). Titration of siRNAs that target $U B C$ mRNA results in minimal viability effects in most $U B B^{\mathrm{WT}}$ lines. The largest effect we observe for $U B C$ knockdown in a $U B B^{\mathrm{WT}}$ cell line occurs in the ovarian line OC316 (HGSOC), for which $5 \mathrm{nM} \mathrm{UBC}$ siRNA produces a 50\% drop in viability at 72 hours. By contrast, in the $U B B^{\text {LO }}$ lines, UBC siRNAs produce a consistent drop in CellTiter-Glo levels that is 20 - to 100-fold below control using $1 \mathrm{nM} \mathrm{UBC}$ siRNA (Figure $5 \mathrm{~A})$. We note that submicromolar exposures as low as $160 \mathrm{pM}$
UBC siRNA produce phenotypes in $U B B^{\mathrm{LO}}$ cell lines that can be greater than 10-fold below control transfected cells. These phenotypes are substantially more potent than those seen with PLK1 knockdown, which produces on average a 5-fold decrease in CellTiter-Glo at $1 \mathrm{nM}$ siRNA. These results highlight the extreme and selective sensitivity of the $U B B^{\mathrm{LO}}$ cells to $U B C$ knockdown. $U B C$ mRNA is reduced $60 \%$ to $80 \%$ in these siRNA titration studies (Supplemental Figure 2A).

We also wanted to assess the long-term effects of knocking down $U B C$ particularly in $U B B^{\mathrm{WT}}$ cells in colony formation assays following siRNA transfection. In these studies, our positive control was PLK1 siRNA, and, as observed above, PLK1 is essential for longterm viability for all cells tested (Figure 5B). Knockdown of $U B C$ prevents colony formation, but selectively in the $U B B^{\text {LO }}$ cells. We also assessed the impact of $U B C$ knockdown by continuously monitoring cell growth and morphology with real-time, live-cell imaging after siRNA transfection and found that depletion of $U B C$ from $U B B^{\mathrm{WT}}$ cells, OC316 (ovarian) and A2058 (melanoma), results in a modest and short-term reduction in growth rate relative to cells treated with control siRNA (Supplemental Figure 2, B and C). By contrast, the $U B B^{\text {LO }}$ cells cannot compensate for the loss of $U B C$ and die.

To consider the short-term impact of ubiquitin depletion on cell cycle progression, $U B C$ was knocked down by siRNA transfection in $2 U B B^{\mathrm{WT}}$ and $2 U B B^{\mathrm{LO}}$ cell lines matched by lineage (skin and ovarian) (Figure 5C). At 30 hours after transfection both of the 

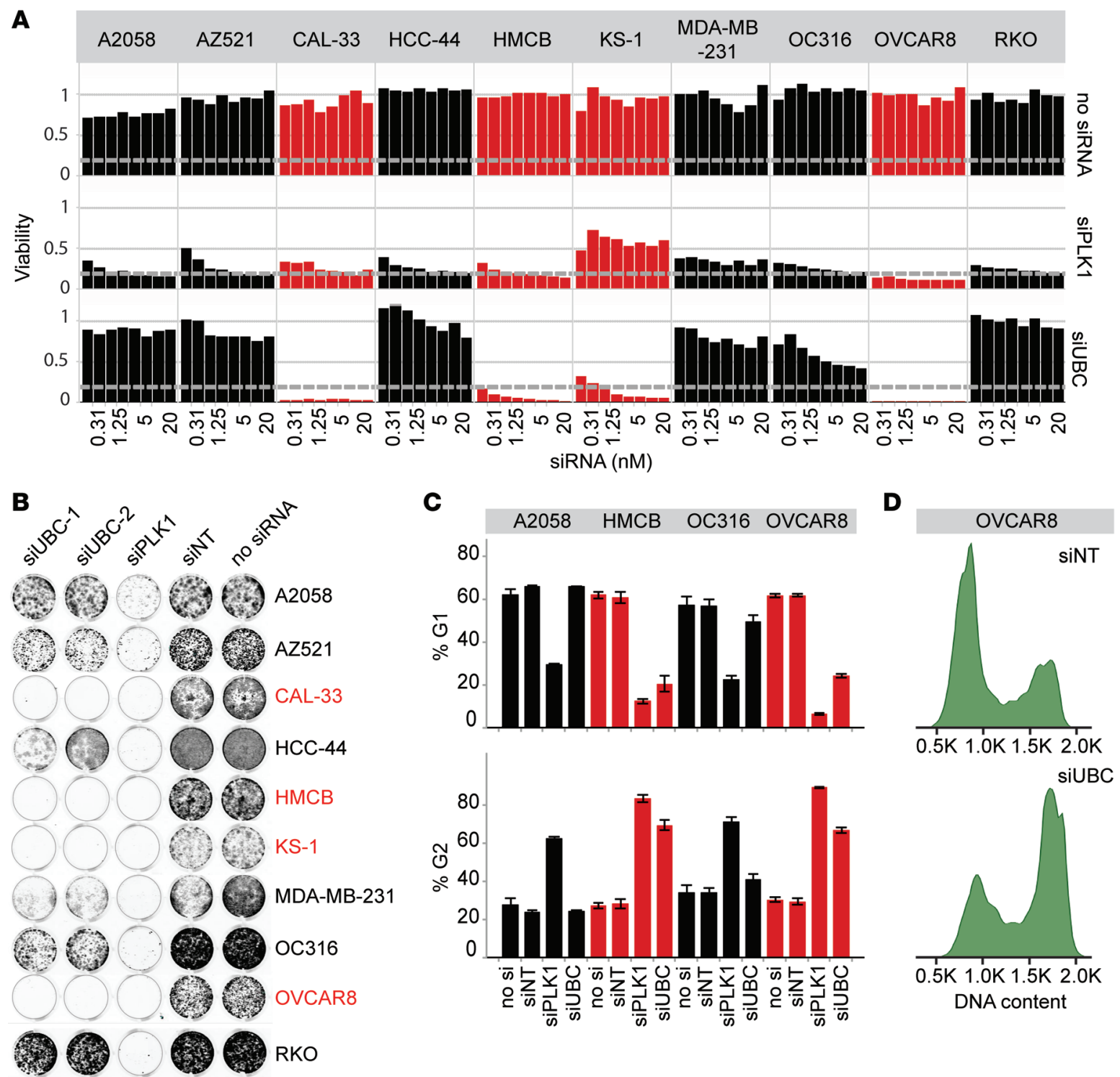

Figure 5. Low UBB expression is sufficient to establish dependency on UBC in diverse-lineage tumors. (A) PLK1 and UBC siRNAs were serially diluted and transfected into $U B B^{W T}$ (black) and $U B B^{L 0}$ (red) cells. Concentrations of target siRNA ranged from 0.16 to $20 \mathrm{nM}$ and included a complementary amount of control nontargeting (NT) siRNA for a final total concentration of $20 \mathrm{nM}$. Dashed line denotes the $10 \%$ viability level (gray). This study was performed 3 times with each point in the siRNA dilution series as a singlicate. (B) Long-term viability in colony formation assays following transfection with siRNAs for PLK1, NT, and 2 independent siRNAs for UBC. After 14 days, cells were fixed and stained with crystal violet. Cell line names shown in red are $U B B^{\llcorner 0}$, black are $U B B^{W T}$. The study was performed multiple times in different configurations. (C) Cell cycle analysis 30 hours after transfection of $U B B^{W T}$ and $U B B^{L 0}$ cells with siRNAs against PLK1, UBC, NT, or no-siRNA controls. Distribution across the cell cycle is shown as percentage $\mathrm{G} 1$ (top) and $\mathrm{G} 2$ (bottom) with SD of biological replicates and repeated twice. Cell cycle data from $U B B^{\mathrm{LO}}$ and $U B B^{\mathrm{WT}}$ cells are shown as red and black bars, respectively. (D) A histogram of DNA content per cell is shown for OVCAR8 transfected with NT siRNA control (top) and UBC siRNA (bottom). This study was performed twice.

$U B B^{\text {LO }}$ lines (HMCB and OVCAR8) show a depletion in cells with G1 DNA content and an accumulation of cells in G2/M. A histogram showing the cell cycle distribution following $U B C$ knockdown in a $U B B^{\mathrm{LO}}$ line is shown (Figure $5 \mathrm{D}$ ). This resembles the effects of PLK1 knockdown previously described to block cells at cytokinesis (25) and which we also observe (Supplemental Figure 2).

Synthetic lethality based on polyubiquitin genes. Our data suggest that the reduced expression of $U B B$ results in a decrease in cellular ubiquitin pools that establishes a dependence on $U B C$.
This hypothesis, if correct, could be directly tested by demonstrating that $U B B$ knockdown in $U B B^{\mathrm{WT}}$ cells is sufficient to make cells dependent on $U B C$ and additionally by rescuing the dependence on $U B C$ in a $U B B^{\text {LO }}$ cell by overexpression of exogenous $U B B$ gene. The first study was conducted by mixing siRNAs together to target transcripts from one or both of these genes and shows that, in $U B B^{\mathrm{WT}}$ ovarian cancer-derived cells, knockdown of either $U B C$ or $U B B$ individually is tolerated; however, silencing of these genes together results in synthetic lethality (Figure 
A

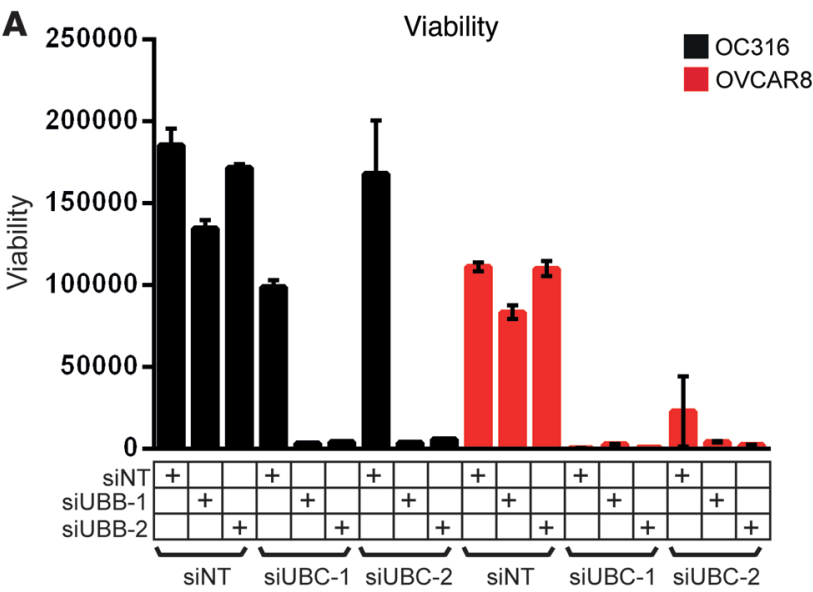

B

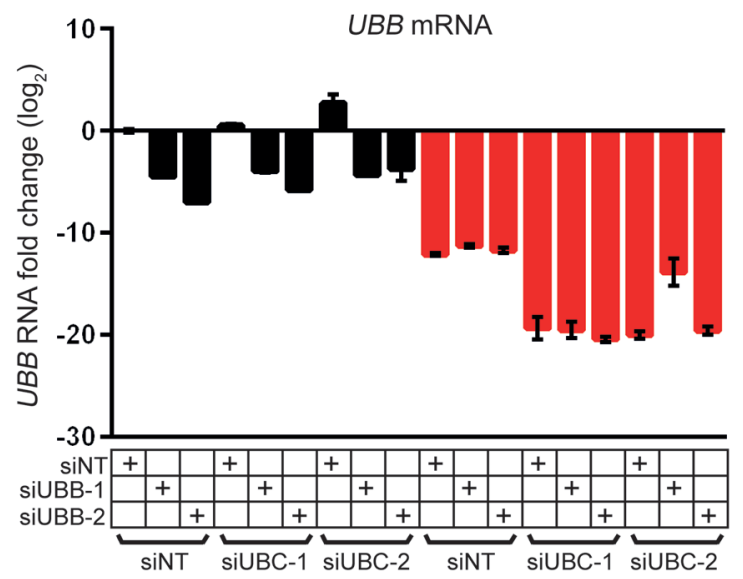

C

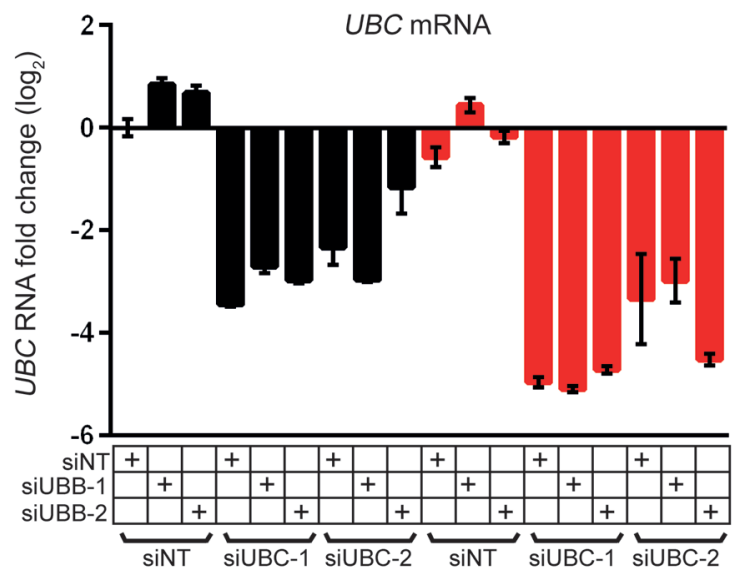

6A). The same results are also obtained in $U B B^{\mathrm{WT}}$ and $U B B^{\mathrm{LO}}$ melanoma cell lines (Supplemental Figure 3). The knockdown of cellular mRNA levels resulting from siRNA treatment is shown for $U B B$ and $U B C$ (Figure 6, B and C).

We also find that the dependency on $U B C$ in OVCAR8 cells, which are $U B B^{\mathrm{LO}}$, can be suppressed by reexpressing $U B B$. In this experiment, cells contain a UBC shRNA that can be induced by doxycycline (Dox) to knock down UBC mRNA and in the presence of Dox, these cells are efficiently eliminated (Figure 6D). However, transfecting these cells with a plasmid that expresses $U B B$ (pUBB) rescues Dox-induced lethality. Expression of plasmid-borne UBB, linked to an antibiotic resistance gene, increases on successive
D

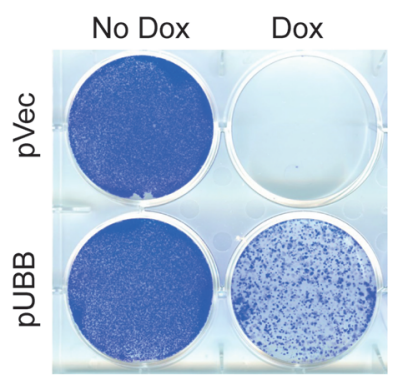

E

Plasmid UBB mRNA

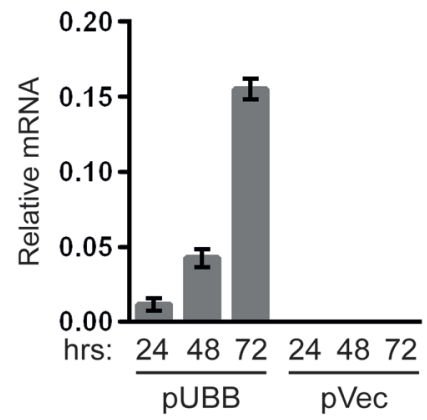

Figure 6. UBB and UBC are a synthetic lethal gene pair. (A) OC316 (black, $U B B^{\mathrm{WT}}$ ) and OVCAR8 (red, $U B B^{\mathrm{LO}}$ ) cells were transfected with a combination of 2 siRNAs for each of UBB and UBC at $5 \mathrm{nM}$ each as shown in the matrix below the figure. Individual siRNAs for UBC or UBB were combined with a nontargeting siRNA (NT) or with each other and viability (CellTiter-Glo assay) was determined 72 hours after transfection. The study was performed twice as biological quadruplicates and the results are shown as mean \pm SEM. (B and $\mathbf{C}$ ) Measurement of siRNA-mediated knockdown of mRNA for (B) UBB and (C) UBC as $\log _{2}$ fold-change relative to OC316 siNT control (far left sample) 24 hours after transfection among biological quadruplicates, with mean \pm SEM and performed twice. (D) Colony formation assays of OVCAR8 containing a doxycycline-inducible (Dox-inducible) UBC shRNA transfected with a neomycin-resistance plasmid encoding UBB ( $p U B B$ ) or the empty vector alone ( $p \vee e c)$. Antibioticresistant cells were seeded into 6-well plates with or without Dox (100 $\mathrm{ng} / \mathrm{ml}$ ) and 7 days later cells were fixed and stained with crystal violet. This study was performed twice. (E) Expression from pUBB was measured on 3 successive days after addition of Dox. Analysis of technical replicates with SD of the mean was performed twice.

days due to survival and outgrowth of antibiotic-resistant, pUBBcontaining cells. (Figure 6E). Together, these studies establish that the primary determinant of lethality to $U B C$ knockdown is the low level of expression from the $U B B$ gene.

Cellular response to a ubiquitin catastrophe. Using an inducible shRNA system enables a regulated and stable approach to study the UBC-mediated synthetic lethal process. Cells transduced to harbor the $U B C$ shRNA were treated with a dose titration of Dox (Figure 7A). Dox produces a viability phenotype in $U B B^{\mathrm{LO}}$ (HMCB) cells with little, or no, effect in $U B B^{\mathrm{WT}}$ (A2058) cells. Knockdown of $U B C$ mRNA is near maximal for both lines at doses above $12.5 \mathrm{ng} /$ $\mathrm{ml}$ after 48 hours of Dox treatment (Figure 7A). 
A

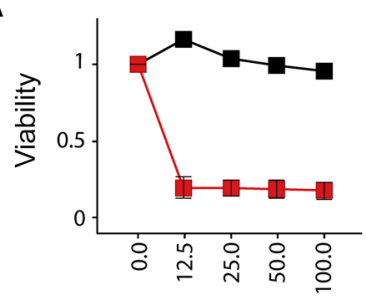

Doxycycline (ng/ml)

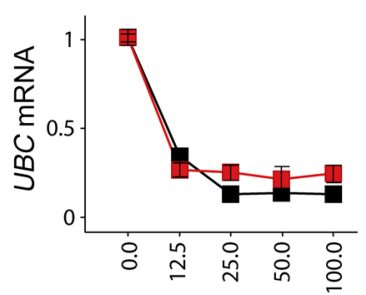

Doxycycline (ng/ml)

B

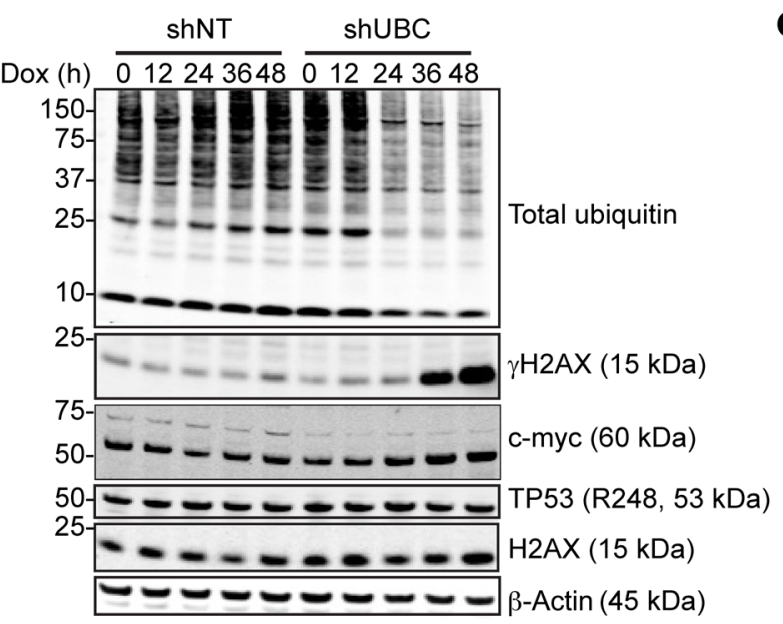

C

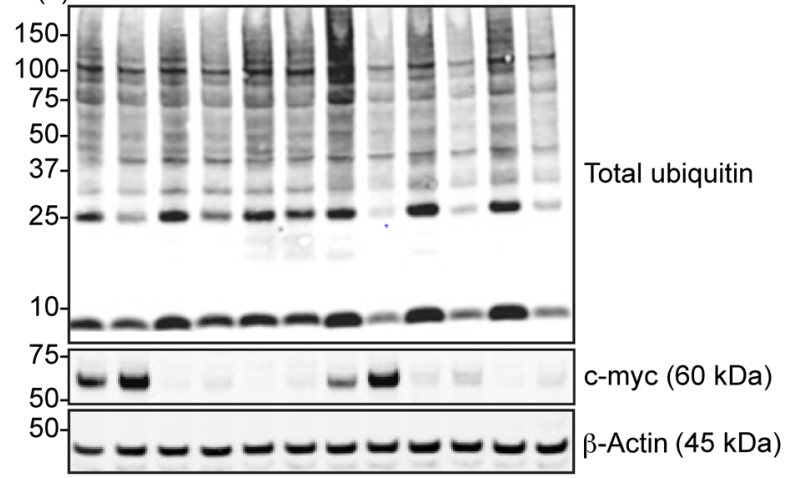

Figure 7. Knockdown of $\boldsymbol{U B C}$ in $\boldsymbol{U B B}^{\mathrm{LO}}$ cells causes a ubiquitin depletion catastrophe. (A) Viability effects at 72 hours (left) in UBB ${ }^{W T}$ (A2058, black) or $U B B^{\mathrm{LO}}$ (HMCB, red) melanoma cells transduced with a doxycycline-inducible (Dox-inducible) shUBC hairpin and exposed to a dose titration of Dox. Studies were performed twice and analysis is replicates with SEM. Knockdown of UBC mRNA is shown at right (48 hours after Dox) and was performed twice with technical replicates and SEM. (B) HMCB $\left(U B B^{\mathrm{LO}}\right)$ cells with shUBC or nontargeting hairpin control shNT were treated with 100 ng/ml Dox, and protein lysates were prepared at the time of Dox addition and at 12, 24, 36, and 48 hours thereafter. Antibodies used for Western blotting were against ubiquitin, $\gamma$-H2AX, c-myc, TP53, total H2AX, and $\beta$-actin. Molecular weight markers (kDa) are shown on the left. (C) Dox (100 ng/ml) was added to cells and lysates prepared at indicated times. Cycloheximide (CHX) was added (10 and $50 \mu \mathrm{g} / \mathrm{ml}) 6$ hours prior to harvesting. Analysis of protein lysates following Dox time course was performed twice each with samples prepared from independent experiments.

The ubiquitin proteins produced from each of the 4 ubiquitinencoding loci are identical, making it impossible to differentiate ubiquitin being made from the $U B B$ or $U B C$ gene, or any of the other ubiquitin-encoding genes. Instead, we are able to assess levels of free ubiquitin as well as protein-ubiquitin conjugates and use this as way to assess levels of cellular ubiquitin (Figure 7B). In this way we monitored time-dependent changes in total ubiquitin levels following induction of the $U B C$ hairpin in $\mathrm{HMCB}\left(U B B^{\mathrm{LO}}\right)$ cells. The level of free ubiquitin and protein-ubiquitin conjugates remains stable for the first 12 hours after addition of Dox but drops precipitously during the next 12-hour period and continues to drop over the remainder of the time course.

The depletion of ubiquitin and ubiquitin-conjugated proteins is specific to $U B C$ knockdown in the $U B B^{\mathrm{LO}}$ cells and is not seen in $U B B^{\text {WT }}$ A2058 cells (X. Liang, unpublished observations). Under these conditions, we observe an increase in c-myc protein after 24 hours as well as an increase in $\gamma-\mathrm{H} 2 \mathrm{AX}$ which appears at 36 hours (Figure 7B). C-myc, an established example of a short-lived protein whose turnover is regulated by ubiquitin-mediated degradation $(26,27)$, provided the insight that the sharp depletion of cellular ubiquitin levels can directly impact protein turnover. We confirm c-myc stabilization in a second $U B B^{\mathrm{LO}}$ cell line following $U B C$ knockdown (Figure 7C).
We also treated cells for 6 hours with cycloheximide (CHX), an inhibitor of protein synthesis, to assess whether the observed increase in steady-state levels of c-myc requires ongoing protein synthesis. CHX treatment reverses c-myc stabilization and suggests that the c-myc turnover rate is slowed but not entirely blocked following ubiquitin depletion and ongoing translation is required to see levels accumulate. These results suggest that the rapid turnover of c-myc protein is sensitive to cellular ubiquitin pool levels and, while not surprising, provides the insight that ubiquitin pool depletion can be affecting diverse systems through decreased protein turnover.

In vivo confirmation of synthetic lethality. These observations encouraged us to consider whether repression of $U B B$ represents a therapeutically exploitable vulnerability in a more physiologically relevant context. For this study, OVCAR8 cells harboring a Dox-inducible UBC hairpin or an siNT were transduced with a constitutively expressed luciferase gene and implanted orthotopically into 24 immunodeficient NOD SCID $\gamma$ (NSG) mice. Tumor-bearing animals were randomly assigned to one of two study arms: 12 would receive Dox by chow and 12 would continue to receive normal chow. On day 37, Dox treatment was initiated at a time when all animals had tumor burdens that were easily seen by luciferase-dependent imag- 
A

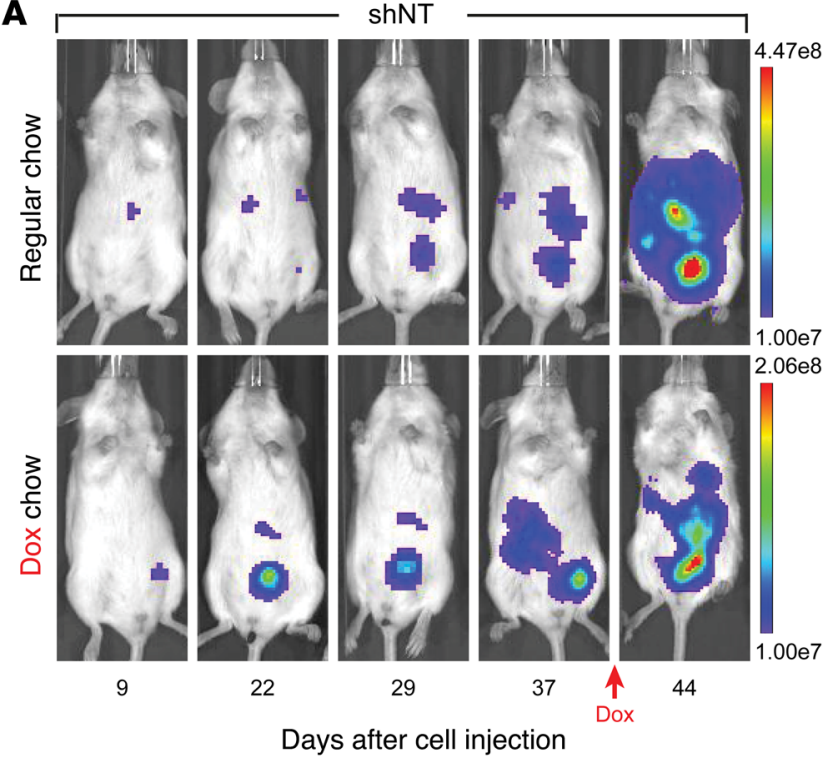

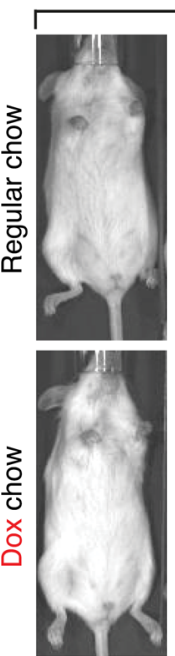

9

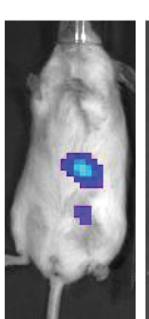

shUBC
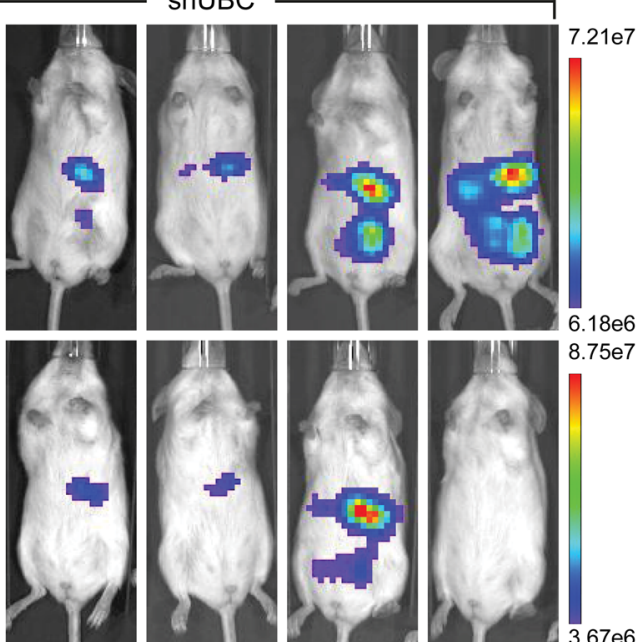

22

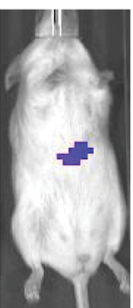

29
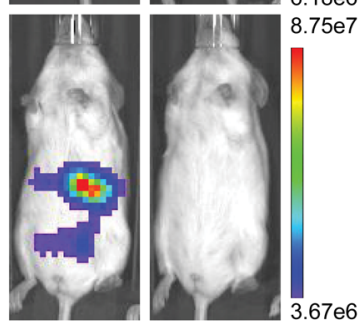

Days after cell injection

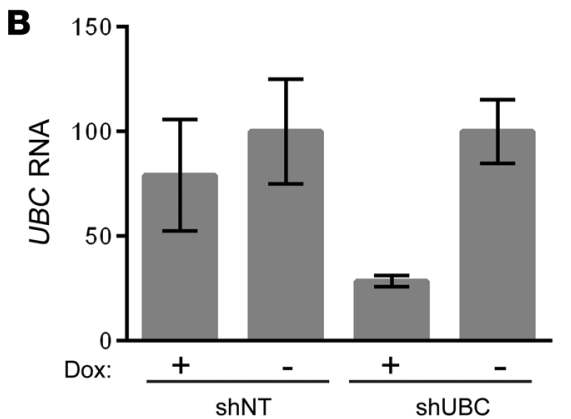

C

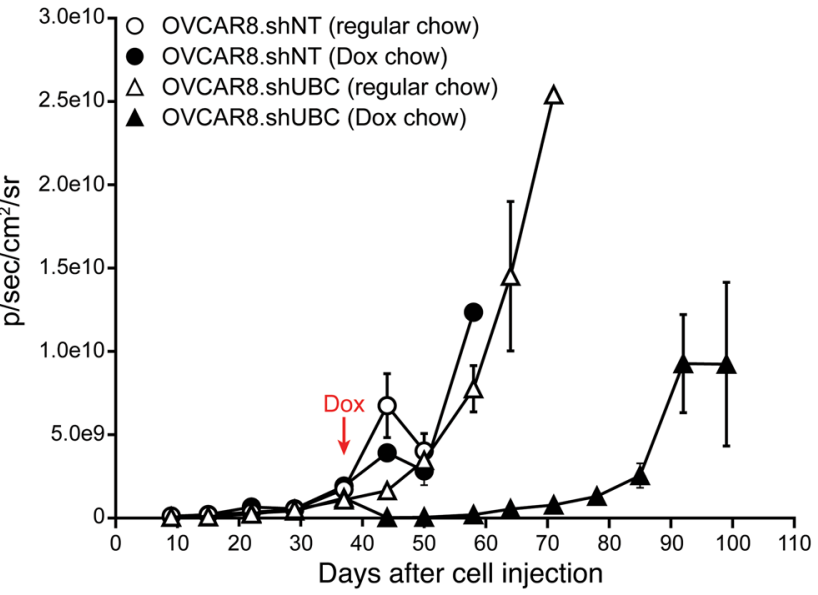

D

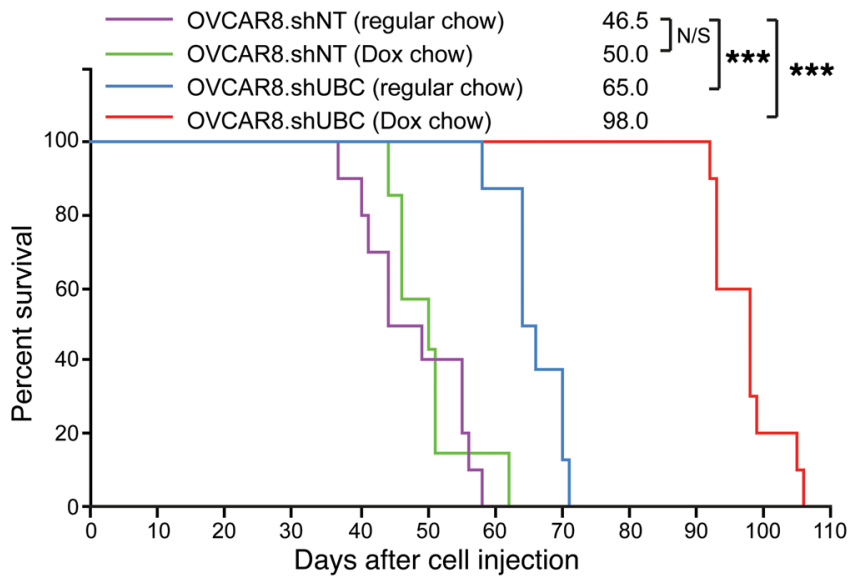

Figure 8. In vivo validation of $\mathbf{U B C}$ synthetic lethality. (A) Luciferized OVCAR8 cells were imaged by bioluminescence on days 9 , $22,29,37$, and 44 after implantation. On day 37 after implantation, half of the mice were transitioned to chow containing doxycycline (Dox). Representative images are shown

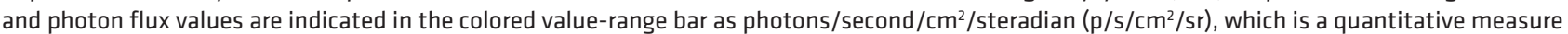
of tumor burden. (B) Pharmacodynamic study of UBC mRNA knockdown 36 hours after the shift to Dox chow. RNA was prepared from multiple tumor nodules from 2 mice per group, and UBC levels were normalized to NT control (no Dox). Error was calculated by SD of technical replicates and the study was done 3 times. (C) Total averaged bioluminescence for 10 mice per arm. Samples derived from shNT with Dox (closed circles) or without Dox (open circles) and shUBC mice with Dox (closed triangles) or without Dox (open triangles). SEM is shown for each time point. (D) Kaplan-Meier plot shows the percentage of mice surviving in each arm of study. All mice in the shNT arm succumb by day 60, and addition of Dox did not affect viability for shNT. Mice bearing tumors with shUBC (blue, red) had increased survival. Statistical significance indicated by $P$ values as follows: not significant (N/S) $=0.61,{ }^{* *} P<0.0002$, ${ }^{* * * *} P<0.0001$. Variation among the groups is substantially less than the difference between the shUBC+Dox and other groups. Tabulation for the survival curves is shown (Supplemental Table 3).

ing (Figure 8A). Within 7 days of starting Dox chow diet, bioluminescence imaging (BLI) values had dropped below detection levels in mice bearing the shUBC-transduced tumor cells (Figure 8A). During the same period BLI values that were monitored biweekly had increased for all other mice. We observed an shUBC-specific decrease in $U B C$ mRNA in these tumors (Figure 8B), and using BLI to monitor tumor burden observed that Dox treatment results in tumor regression (Figure $8 \mathrm{C}$ ). We conclude that the dependency on $U B C$ in the $U B B^{\mathrm{LO}}$ cells is also relevant in the in vivo setting. 
We further used this model to determine the survival benefit of targeting $U B C$ in these cells. Animals in the control arms developed symptoms of liver failure due to tumor cell infiltration and rapidly succumbed to disease, with median survival around 50 days (Figure 8D). By contrast, mice implanted with OVCAR8 shUBC and continuously fed Dox chow had durable remissions and substantial long-term survival benefit. In light of these responses, it is noteworthy that shUBC tumor cells go on to develop resistance in mice treated with Dox (Figure 8C) and the mice eventually die, with a median survival of nearly 100 days after implant.

The resistant tumors provided an opportunity to determine whether there are potential on-pathway adaptions, such as derepression of $U B B$, that rescue lethality by restoring ubiquitin levels. Dox-resistant tumors were removed from 5 mice and ex vivo growth was established to potentially gain insight into the resistance mechanism. Our analysis revealed that resistance was the result of ineffective $U B C$ knockdown rather than reexpression of $U B B$. In these studies, we found that all Dox-resistant lines still retained a dependency on $U B C$ and were sensitive to $U B C$ knockdown by siRNAs (Supplemental Figure 4). The likely explanation for these observations is that a small number of cells implanted into mice are not competent to express the $U B C$ hairpin and continue to proliferate though the treatment with Dox that otherwise produces deep remissions through the loss of cells expressing shUBC. The nonresponder cells eventually overtake the animal, necessitating euthanasia. It is noteworthy that despite strong selective pressure, we did not find evidence for $U B B$ reexpression in any of the Dox-resistant cultures, suggesting that silencing at this locus is governed by a potent, heritable, and stable mechanism.

\section{Discussion}

Our analysis of TCGA identifies low $U B B$ expression as a frequent alteration in gynecological cancers linked to poor survival, and we establish a model to consider what, if any, therapeutic responses could be achieved should it be possible to target cancers with this lesion.

Why would reduced levels of $U B B$ be selected for in cancer? Ubiquitin is essential, yet cells produce ubiquitin through multiple genetic and regulatory strategies that makes it possible to tune levels of ubiquitin pools for survival and likely other developmental decisions. The yeast polyubiquitin gene, UBI4, is not required for vegetative growth but is needed for stress response and for sporulation (28). Experiments looking for genes regulating fitness adaptability identified loss-of-function mutations in UBR1, an E3 ubiquitin ligase involved in $\mathrm{N}$-end rule degradation (29), and genes involved in protein degradation suppress growth defects associated with mutational burdens presumably by stabilizing hypomorphic proteins (30). The role for ubiquitin may be as a pleiotropic regulator and UBI4 hypomorphic alleles produce spontaneous phenotypic variants in hyphal development and morphogenesis in candida (31).

A genomics approach termed CYCLOPS identified singlecopy deletions of PSMC2, a component of the proteasome, as a recurrent event in a subset of HGSOC (32). Both hemizygous $P S M C 2$ copy number loss and transcriptional repression of $U B B$ might serve to optimize cell fitness by tuning protein fate in a way that stabilizes prosurvival proteins during tumorigenesis (33).
However, the loss of $U B B$ leads to reductions in the ubiquitin pool, the benefit of which may ultimately be offset by the cost associated with shifting the cell closer to a minimal ubiquitin threshold.

Global loss of ubiquitin is lethal, as shown using temperaturesensitive alleles of a ubiquitin-activating enzyme (ts85) that leads to depletion of free ubiquitin, ubiquitin-protein conjugates, stabilization of short-lived proteins, and a transient accumulation in G2 phase prior to cell death (34). This is consistent with our observations of c-myc stabilization and an expanded G2 population shortly following $U B C$ knockdown in $U B B^{\mathrm{LO}}$ cells.

A role of ubiquitin in chromatin modification and the DNA damage response is well described $(35,36)$. Induction of $\gamma-\mathrm{H} 2 \mathrm{AX}$ in the context of acute ubiquitin depletion is consistent with previous observations that $U B B$ and $U B C$ suppress spontaneous DNA damage during otherwise unperturbed growth (37) and are required for normal 53BP1 foci formation following irradiation (38). However $\gamma$-H2AX also occurs by other, DNA-independent stresses including heat shock (39), and following UBC knockdown may be unrelated to defective DNA repair and instead could result from DNA cleavage during apoptosis (40).

In mammals, the polyubiquitin genes $U B B$ and $U B C$ play different roles in development. $U b c$-null mice die in mid-gestation due to disruption of fetal liver function $(41,42)$, while mice that are homozygous null for $U b b$ are viable but sterile (43). Polyubiquitin genes have been found to be required for meiosis in yeast, fly, and mammals (44-46), suggesting that levels of ubiquitin play specific developmental roles. How this relates to the depletion of ubiquitin by silencing of $U B B$ during tumorigenesis remains unclear. Expression of the $U B B$ gene is otherwise best described as a housekeeping gene; when examined across 30 tissue types, it is less variable than GAPDH, and there is no tissue in which $U B B$ appears transcriptionally inactive (Supplemental Figure 1B). These results do not provide insight to help explain the incidence of $U B B$ silencing in gynecological tissues and leaves open the possibility that $U B B$ repression is a consequence of alterations in ovarian surface and fallopian epithelium (47-49). All 3 tumor types with a high incidence of $U B B$ silencing are derived from Müllerian epithelium, a finding that should prompt follow-up studies to consider $U B B$ expression and promoter methylation status in these cells (50). Therefore, although ovarian cancer arises from tubal epithelium, normal ovary where $U B B$ expression is normal was employed as a useful general reference point.

Repression of $U B B$ is unlikely to be a spurious, passenger lesion. This locus is vulnerable to DNA methylation and transcriptional silencing by a locally restricted mechanism that does not extend to the closest flanking genes, CENPV or TRPV2, which are located just $28 \mathrm{~kb}$ to the $5^{\prime}$ side and $35 \mathrm{~kb}$ to the $3^{\prime}$ side, respectively (Supplemental Figure 5). We also note that $U B B$, located on chromosome 17p (17p11.2), is rarely deleted. Lastly, there is no evidence for $U B C$ silencing, although previous knockout studies as well as work herein show that diverse cell types can tolerate silencing of $U B B$ or $U B C$ separately $(41,42,51)$.

Together, these findings provide a rationale for the development of a medicine targeting $U B C$ at the mRNA level that might include siRNA therapeutics $(52,53)$, antisense oligonucleotides $(54,55)$, or other approaches that reduce ubiquitin levels. In our studies, we see little to no toxicity caused by $U B C$ silencing in 
cells with normal expression of $U B B$, although tolerance for systemic silencing of $U B C$ remains untested. While acute knockdown of $U B C$ in cells can lead to a transient proliferative delay of cell lines in vitro, $\mathrm{Ubc}^{+/-}$heterozygous mice appear normal (41). These observations warrant further studies to exploit this therapeutic opportunity for a large population of cancer patients lacking actionable targets for precision medicine.

Our findings provide a potentially new perspective on previous reports of synthetic lethality among functional paralogs involved in likely compensatory roles in fitness. These include ARID1A and $A R I D 1 B$ (56), SMARCA2 and SMARCA4 (57), and most recently CBP and EP3OO (58). Although the roles of these genes in tumorigenesis are similarly unclear, these examples of synthetic lethality can now be expanded to include $U B B$, a ubiquitin-encoding gene that serves as a biomarker for a clinically aggressive HGSOC subtype, and its paralog $U B C$. Our observation offers insight for the development of targeted agents in a newly defined patient population.

\section{Methods}

Cell culture. All cell lines were authenticated using an Affymetrix SNP 6.0 array (Asuragen) and compared to reference genomes in CCLE (23) and found to be negative for mycoplasma using the MycoAlert kit (Lonza, LT07). Growth of all lines was at $37^{\circ} \mathrm{C}$ with $5 \% \mathrm{CO}_{2}$ in the corresponding media: CAL33, HMCB, and A2058 in DMEM (Gibco); DEL, HCC-44, OC316, OVCAR8, MDA-MB-231, and NCI-1299 in RPMI (Gibco); JHOM1 in DMEM:F12 (Gibco); KYSE150 in RPMI:F12 (Gibco); KS-1, AZ521, and OVK18 in EMEM (ATCC); and RKO in McCoy's 5A (Gibco). During authentication, we determined that AZ521 is commonly misidentified as HuTu80; however, the cells in our study were confirmed as AZ521 against the CCLE reference. All media were supplemented with $10 \%$ FBS (Hyclone, SH30071.03). The tet-on promoter was induced with $100 \mathrm{ng} / \mathrm{ml}$ Dox (MilliporeSigma, T7660). For colony formation, cells were fixed and stained using crystal violet solution $(0.2 \% \mathrm{w} / \mathrm{v}$ crystal violet [MilliporeSigma, C0775], $4 \% \mathrm{v} / \mathrm{v}$ formalin [Thermo Fisher Scientific, F79-500] in PBS) for 15-30 minutes and washed 3 times with distilled water.

mRNA expression level. RNA was extracted using the RNeasy Kit (Qiagen, 74181) or the TurboCapture 96 mRNA kit (Qiagen, 72251). cDNA was reverse transcribed using the High-Capacity cDNA Reverse Transcription Kit (Applied Biosystems, 4368814) or Cells-to-Ct RT reagents (Applied Biosystems, 4391852C). mRNA levels were determined by Taqman quantitative PCR (qPCR) using an ABI7900HT Fast Real-Time PCR instrument (Applied Biosystems) with the following gene-specific probe sets purchased from Applied Biosystems: UBA52 Hs03004331_g1, RPS27A Hs01923841_uH or Hs01577583_g1, UBB Hs00430290_m1, UBC Hs01871556_s1 or Hs00824723_m1, and PLK1 Hs00153444_m1. Data were normalized using an internal control gene, either GAPDH (Thermo Fisher Scientific, 4352934E) or $\beta$-actin (Applied Biosystems, Hs99999903_m1). Relative quantification of expression levels (RQ) was calculated using the $2^{-\Delta \Delta C t}$ method or relative quantification (59). Expression in tumor tissue involved multiple nodules per mouse which were removed and stored in RNAlater. mRNA was extracted with TRIzol (Ambion, 15596-018) and isolated using the RNeasy Kit. Samples were subject to reverse transcription and amplified by Taqman. Results were analyzed individually and subsequently combined as a group. Four to 5 nodules were isolated from each mouse, and 2 mice were included from each group.
To restrict the analysis to the human cells in the sample, results were normalized to human $G A P D H$ by the $\triangle \triangle \mathrm{CT}$ method. $U B C$ levels were then normalized to the shNT-no-Dox sample. mRNA values for genes proximal to UBB on chromosome 17 were analyzed by the $\triangle \mathrm{CT}$ method, which was normalized to an internal control, $\beta$-actin.

siRNA transfection. UBC siRNAs were obtained from Dharmacon (J-019408-05, J-019408-06, J-019408-07 [UBC-1], and J-019408-08 [UBC-2]) or Qiagen (SI00754271, SI03048423, and SI05071444). UBB siRNAs were obtained from Qiagen S103107328 (UBB-1) and Dharmacon J-03382-07 (UBB-2). siNT was purchased from Dharmacon (D-001810-01-05), and PLK1 siRNA was from Qiagen (SI02223837). siRNAs were resuspended to a final stock concentration of $20 \mu \mathrm{M}$ in nuclease-free water. For titration studies, a dilution of siRNA (OptiMEM I) was prepared at 10 $\times$ final concentration. Dharmafect-1 (Dharmacon, T-2001) was diluted 1:40 in OptiMEM I for a 1:400 final concentration. To $10 \mu \mathrm{l}$ of diluted siRNAs per well in 96-well plates, 10 $\mu$ of diluted Dhamafect- 1 was added followed by 10,000 cells in 80 $\mu \mathrm{l}$ media. One hundred microliters of CellTiter-Glo (Promega, G7573) was added at 72 hours and relative light units (RLU) were measured using the EnVision plate reader (PerkinElmer). The Incucyte (Essen Bioscience) was used for microscopy assays. Colony formation assay (CFA) results were reproducible with 6 independent UBC siRNAs.

Matrix siRNA studies included 2 UBC siRNAs and 2 UBB siRNAs and an siNT at a final concentration of $10 \mathrm{nM}$ for each siRNA. mRNA levels for $U B B$ and $U B C$ were assessed 24 hours after transfection. Caspase-3/7 was detected using an IncuCyte Green Apoptosis assay 4440 (Essen Biosciences) (24).

Cell cycle analysis. Cells were plated into 6-well plates $\left(0.5 \times 10^{6}\right.$ per well) as biological replicates and transfected the next day. Thirty hours later, cells were trypsinized, washed with cold PBS, fixed in 70\% ethanol/water, pelleted, and resuspended in a staining solution containing 0.1\% Triton X-100, $200 \mu \mathrm{g} / \mathrm{ml}$ DNase-free RNase A (Thermo Fisher Scientific, 12091-021), and $20 \mu \mathrm{g} / \mathrm{ml}$ propidium iodide (Roche, 11348639001). DNA content data were acquired from 10,000 cells using a BD Fortessa flow cytometer at $488 \mathrm{~nm}$. Cell cycle distribution analysis was performed with FlowJo software (www.flowjo.com).

Data acquisition and in silico analysis. The results included are in part based on data generated by the TCGA Research Network (http:// cancergenome.nih.gov). TCGA Level_3 RNASeq V2 Illumina HiSeq and Infinium Human DNA Methylation (27 and 450; two different methylation array chips) data were downloaded via the TCGA bulk download site (https://tcga-data.nci.nih.gov/tcga/). TCGA gene-level RSEM values (60) reflect gene expression abundance (transcript reads per length) and methylation $\beta$ values (22) were collated with patient annotation details using python v3.4. Analysis of nontumor samples from the GTEx Consortium (61) involved gene-level RPKM values that were collated and summarized using python v3.4. Determination of sample outliers for the $U B C / U B B$ ratio (calculated in Spotfire v6.5) was defined as 1.25, based on the upper inner fence of the ratio, a standard statistical measure of outlier status, defined as $Q 3+(1.5 \times$ $I Q)$, where $I Q$ is the interquartile range. Kernal density estimates were calculated in R v3.2.0 using the mclust package (62), where Bayesian information criterion optimally supports the presence of 2 Gaussian components for UBB expression in HGSOC tumors with singular population estimates for the other ubiquitin genes. Kaplan-Meier survival estimates were generated in R v3.2.0 using the survival package (63), with HGSOC tumors stratified by the first quartile of $U B B$ expression 
(low $=\log _{2}[U B B] \leq Q 1$, high $\left.=\log _{2}[U B B]>Q 1\right)$. Cancer cell line encyclopedia levels of expression and copy number of the ubiquitin genes $U B B$, $U B C, U B A 52$, and RPS27A as well as the control GAPDH were derived from GC-RMA-normalized Affymetrix Human Genome U133 Plus 2.0 microarray measurements available at www.broadinstitute.org/ccle. One cell line, SNU283, appears to have a biallelic deletion of the chromosome 17 region encompassing $U B B$. We were unable to establish these cells in culture to confirm their identity as well as expression data and consequently these were excluded from our analysis.

shRNA cloning and viral packaging. shUBC (target sequence CGAGAACGTCAAAGCAAAGAT) and shNT (TGGTTTACATGTCGACTAA) forward and reverse complementary oligonucleotides were purchased from IDT, annealed following denaturing at $95^{\circ} \mathrm{C}$ for $2 \mathrm{~min}-$ utes, and then allowed to cool to room temperature in annealing buffer (10 mM Tris 8.0, $50 \mathrm{mM} \mathrm{NaCl}, 1 \mathrm{mM}$ EDTA) and ligated into the Dox-inducible plasmid pRSI16-puro at a unique BbsI site (Cellecta). Newly constructed plasmids were confirmed by sequencing with the U6S primer (CAAGGCTGTTAGAGAGATAATTGGA). shRNA plasmid vector was cotransfected into 293T cells along with the packaging and envelope vectors $\Delta 8.9$ and VSV-G at a 6:6:1 molar ratio using Lipofectamine 2000 (1:500 dilution, Thermo Fisher Scientific). Supernatant was collected 48 hours later, and viral titer was determined by following infection in the presence of $5 \mu \mathrm{g} / \mathrm{ml}$ polybrene. After 24 hours, media were changed and viability was assessed in replica cultures grown for 7 days in the absence or presence of $1 \mu \mathrm{g} / \mathrm{ml}$ puromycin.

Rescue of UBC-knockdown toxicity by expression of $U B B$. UBB cDNA was purchased (Thermo Fisher Scientific, IOH56688), and its sequence was validated by primer walking and was transfected into OVCAR8 cells harboring shUBC using Lipofectamine LTX with PLUS Reagent (Thermo Fisher Scientific, 15338030). pDEST40 empty vector $(\mathrm{pVec})$ was used as control. Twenty-four hours later, G418 (200 $\mu \mathrm{g} /$ $\mathrm{ml}$, Gibco, 11811-023) was added for 7 days. G418-resistant cells were maintained in RPMI supplemented with $10 \%$ tetracycline-free FCS, puromycin $(1 \mu \mathrm{g} / \mathrm{ml})$, and $\mathrm{G} 418(200 \mu \mathrm{g} / \mathrm{ml})$. Cells were seeded into four 6-well plates (200,000 cells/well) with media containing or lacking $100 \mathrm{ng} / \mathrm{ml}$ Dox to induce shRNA expression, and colony formation was performed as described above. To detect $U B B$ transcripts under the control of the CMV promoter in the pDEST40 vector, a CMVUBB probe set was designed to anneal to the junction portion of the CMV promoter and UBB cDNA using the IDT PrimerQuest Real-Time PCR Design tool and included the forward primer GACCCAAGCTGGCTAGTTAAG, reverse primer CCTCAAGGGTGATGGTCTTG, and probe FAM/AGGCACCATGCAGATCTTCGTGAA/TAMSp). To measure expression of $U B B$ coming from the plasmid, RNA was isolated 24, 48, and 72 hours after addition of Dox.

Western blotting. HMCB (shUBC or shNT or shPLK1) and A2058 (shUBC) cells were plated into 6-well plates (1,000,000 cells/well), and Dox (100 ng/ml) was added the next day. Cells were harvested at 12-hour intervals after addition of Dox in RIPA buffer (Teknova, R3792) supplemented with Halt protease inhibitor cocktail (Thermo Fisher Scientific, 1861278) and Halt phosphatase inhibitor (Thermo Fisher Scientific, 1861277). The following antibodies (Cell Signaling Technology) were used for protein biomarker detection: ubiquitin (catalog 3933), c-myc (catalog 5605), phospho-H2A.X-S139 (catalog 2577), $\alpha$-tubulin (catalog 3873), and histone H2A.X (catalog 7631). Secondary antibodies were purchased from Odyssey (catalog 926-32211 and 926-68020), and fluorescence was detected using an Odyssey LI-COR.
Mouse study. OVCAR8 cells harboring shRNAs were luciferized by infecting with pMMP-LucNeo retrovirus (64) and selected with $100 \mathrm{ng} / \mathrm{ml} \mathrm{G} 418$. The xenograft model was established by intraperitoneal injection of $2 \times 10^{6}$ OVCAR8.shControl or OVCAR8.shUBC cells in 6-week-old female NSG (NOD.Cg-Prkdc ${ }^{\text {scid }} \mathrm{Il}_{2} \mathrm{rg}^{\mathrm{tm} 1 \mathrm{Wil}} / \mathrm{SzJ}$, stock number 005557, The Jackson Laboratory). Tumor growth was monitored weekly using the IVIS Spectrum In Vivo Imaging System (PerkinElmer), starting at day 9 after cell injection. Briefly, mice were injected subcutaneously with $75 \mathrm{mg} / \mathrm{kg}$ D-luciferin potassium salt (Promega, E1605) in sterile PBS and anesthetized with 2\% isoflurane in medical air. Serial bioluminescence images were acquired using the automated exposure setup. The peak bioluminescence signal intensity within selected regions of interest (ROIs) was quantified using Living Image Software (PerkinElmer) and expressed as photon flux (photons/second $/ \mathrm{cm}^{2} /$ steradian). Representative planar bioluminescence images were displayed with indicated adjusted minimal and maximal thresholds. Mice were randomized into 2 groups per cell line, for a total of 4 groups ( $n=12$ per group; 10 for efficacy and 2 for pharmacodynamics), using the overall tumor burden as measured by BLI. The recruited animals were randomized into treatment groups with similar BLI signal means, indicative of comparable disease burden. The investigators were not blinded to allocation during experiments and outcome assessment. Mean BLI for each group of 10 animals was calculated, and error was determined by SEM. Median survivals were determined using the method of Kaplan and Meier. A log-rank (Mantel-Cox) test was used to calculate $P$ values derived from statistical analysis of the Kaplan-Meier survival curves. $P$ values less than 0.05 were considered to be statistically significant. Analysis was performed with Prism 6 (GraphPad Software, Inc.). Standard chow was replaced with Dox chow (2,000 ppm) for one group in each cell line at day 37 after cell injections and continued until study completion. All animals were further followed for survival. When signs of morbidity occurred (such as weight loss, jaundice, and/or ascites-associated bloating of the abdomen), the affected animal was euthanized according to the 2013 AVMA (American Veterinary Medical Association) guidelines. The animals were imaged weekly to assess tumor establishment and progression. As tumor burdens were comparable at 5 weeks after cell injection, all animals were enrolled in treatment groups. A small number of animals developed palpable subcutaneous tumor, and were thus excluded from the study. As a result final efficacy study included 7 to 10 animals per group. On some occasions, the tumor lesions were excised, confirmed by ex vivo BLI, and stored in RNAlater (Ambion, AM7020) for further analysis. Tissue was homogenized in TRIzol solution, and RNA was isolated using the RNeasy Kit (Qiagen, 74106).

Resistance studies. tetR mRNA expression level was detected with a custom qPCR probe set (forward primer GCCTACAGAGAAGCAGTATGAG, reverse primer AGAGGGCATACAAGGCATTT, and probe FAM/AAGCCTTGTTGGCACAGGAATGC/TAMSp). Hairpin expression was measured using a probe designed and synthesized by Exiqon based on the shUBC target sequence CGAGAACGTCAAAGCAAAGAT. miRNA was isolated using a miRNeasy Mini Kit (Qiagen, 217004), and cDNAs were synthesized using a miRCURY LNA Universal RT microRNA PCR Starter Kit (Exiqon, 203351). Briefly, a poly-A tail added to the mature shRNA transcript was used as an anchor for a poly-T primer to convert this into cDNA, which was amplified using template-specific and LNA-enhanced forward and reverse primers and product was detected by SYBR Green fluorescence and 
quantified by Applied Biosystems 7900 real-time PCR. Cells were cultured in RPMI containing $10 \%$ tet-free FCS and $1 \mu \mathrm{g} / \mathrm{ml}$ puromycin. Cells were transfected with siRNAs at a final concentration of $5 \mathrm{nM}$ and $U B C$ mRNA was measured 24 hours after transfection.

Statistics. Included in the description of TCGA expression and methylation data, the Pearson correlation analysis of subtype-specific methylation and expression of $U B B$ is as follows (associated Pearson $R^{2}$ and $P$ values): immunoreactive $=-0.66,6.8 \mathrm{e}^{-21} ;$ mesenchymal $=$ $-0.69,2.5 \mathrm{e}^{-14} ;$ differentiated $=-0.74,1.6 \mathrm{e}^{-25} ;$ and proliferative $=-0.84$, $2.3 \mathrm{e}^{-46}$. The significance threshold was set at the $99 \%$ confidence interval $(P$ value $<0.01)$.

Kaplan-Meier survival estimates for TCGA HGSOC were generated in R v3.2.0 using the survival package (63), with ovarian tumors stratified by the lowest quartile of $U B B$ expression in HGSOC $\left(\log _{2}[U B B] \leq Q 1\right)$ plotted against samples with UBB expression $\log _{2}(U B B)>Q 1$. Survival curve differences for low and high UBB expression groups were compared using the Mantel-Haenszel logrank test (65). The low-expressing group was determined to have significantly poorer outcomes. The $\chi^{2}=6.06$ on 1 degree of freedom, $P$ value $=0.0138$. The significance threshold was set at the $95 \%$ confidence interval $(P$ value $<0.05)$.

Gaussian mixture model-based kernel density estimates were calculated in R v3.2.0 using the mclust package (62), where Bayesian information criterion optimally supports the presence of 2 Gaussian components for $U B B$ expression in ovarian tumors (Figure $2 \mathrm{~B}$ ) with singular population estimates for the other ubiquitin genes.

Determination of sample outliers for the $U B C / U B B$ ratio (calculated in Spotfire v6.5) was defined as 1.25, based on the upper inner fence of the ratio, a standard statistical measure of outlier status, defined as $Q 3+(1.5 \times I Q)$.

For the survival study OVCAR8 xenograft tumor model, the log-rank Mantel-Cox test was used to compare survival curves. The significance threshold was set at the $99.99 \%$ confidence interval $(P$ value $<0.0001)$.

Study approval. The present studies in animals were reviewed and approved by the Dana-Farber Cancer Institute Institutional Animal Care and Use Committee (IACUC), Boston, Massachusetts, USA.

\section{Author contributions}

ATK, JJ, MSG, and WCF conceived of the experiments. ATK, SG, MSG, and WCF prepared the manuscript. XL designed and performed the inducible shRNA studies. MSG, DMB, EG, PCG, QDN, PTK, and RJD designed and performed the mouse studies. DMB and PCG acquired and analyzed the mouse data. DMB and XL performed RNA studies from tumor grafts and studies involving doxycycline-resistant tumors. SG acquired data and performed the analysis of patient tumor-derived data from The Cancer Genome Atlas project. XL, ATK, SS, FH, FS, and CB performed the experiments.

\section{Acknowledgments}

The authors would like to acknowledge the contributions of John Koschwanez (Novartis Institutes for Biomedical Research [NIBR]), Aaron Jaffe (NIBR), and Kris Wood (Duke University) for comments on the manuscript; Craig Mickanin and Frank Girardi (NIBR) for providing reagents used in the siRNA studies; and Jeff Porter, John Tallarico, Alan Buckler, and Joe Loureiro (NIBR) for their support and encouragement. The authors would also like to acknowledge the Bioinformatics team in the Oncology group at NIBR for development of the CCLE data portal used in Figure 3, A and B. Hong Tiv (Dana-Farber Cancer Institute) provided assistance with the mouse work and Christy Fryer shared the pMMPLucNeo retrovirus. M.S.G. is a Liz Tilberis Scholar of the Ovarian Cancer Research Fund Alliance. The results shown here are in part based on data generated by the TCGA Research Network: http:// cancergenome.nih.gov. Anonymous reviewers are recognized for sharing their expertise on gynecological cancer and comments that significantly improved the content of the manuscript.

Address correspondence to: Michael S. Goldberg, Dana-Farber Cancer Institute, 450 Brookline Ave., Boston, Massachusetts 02215, USA. Phone: 617.909.8181; Email: michael_goldberg1@dfci.harvard. edu. Or to: William C. Forrester, Chemical Biology and Therapeutics, Novartis Institutes for Biomedical Research, 500 Technology Square Ave., 8B.002.04.A, Cambridge, Massachusetts 02139, USA. Phone: 617.871.7265; Email: william.forrester@novartis.com.
1. Pagliarini R, Shao W, Sellers WR. Oncogene addiction: pathways of therapeutic response, resistance, and road maps toward a cure. $E M B O$ Rep. 2015;16(3):280-296.

2. McLornan DP, List A, Mufti GJ. Applying synthetic lethality for the selective targeting of cancer. NEngl JMed. 2014;371(18):1725-1735.

3. Bitler BG, et al. Synthetic lethality by targeting EZH2 methyltransferase activity in ARID1Amutated cancers. Nat Med. 2015;21(3):231-238.

4. van den Bent MJ. Interobserver variation of the histopathological diagnosis in clinical trials on glioma: a clinician's perspective. Acta Neuropathol. 2010;120(3):297-304.

5. Verhaak RG, et al. Integrated genomic analysis identifies clinically relevant subtypes of glioblastoma characterized by abnormalities in PDGFRA, IDH1, EGFR, and NF1. Cancer Cell. 2010;17(1):98-110.

6. Parsons DW, et al. An integrated genomic analysis of human glioblastoma multiforme. Science. 2008;321(5897):1807-1812.
7. Dang L, Yen K, Attar EC. IDH mutations in cancer and progress toward development of targeted therapeutics. Ann Oncol. 2016;27(4):599-608.

8. Loehrer PJ, Einhorn LH. Drugs five years later. Cisplatin. Ann Intern Med. 1984;100(5):704-713.

9. Vaughan S, et al. Rethinking ovarian cancer: recommendations for improving outcomes. Nat Rev Cancer. 2011;11(10):719-725.

10. Rodriguez-Freixinos V, Mackay HJ, Karakasis K, Oza AM. Current and emerging treatment options in the management of advanced ovarian cancer. Expert Opin Pharmacother. 2016;17(8):1063-1076.

11. Narod S. Can advanced-stage ovarian cancer be cured? Nat Rev Clin Oncol. 2016;13(4):255-261.

12. Coleman RL, Monk BJ, Sood AK, Herzog TJ. Latest research and treatment of advanced-stage epithelial ovarian cancer. Nat Rev Clin Oncol. 2013;10(4):211-224.

13. Verhaak RG, et al. Prognostically relevant gene signatures of high-grade serous ovarian carcinoma. J Clin Invest. 2013;123(1):517-525.

14. Zhang $\mathrm{H}$, et al. Integrated proteogenomic char- acterization of human high-grade serous ovarian cancer. Cell. 2016;166(3):755-765.

15. Winterhoff B, et al. Molecular classification of high grade endometrioid and clear cell ovarian cancer using TCGA gene expression signatures. Gynecol Oncol. 2016;141(1):95-100.

16. Kim G, et al. FDA approval summary: olaparib monotherapy in patients with deleterious germline BRCA-mutated advanced ovarian cancer treated with three or more lines of chemotherapy. Clin Cancer Res. 2015;21(19):4257-4261.

17. Lord CJ, Tutt AN, Ashworth A. Synthetic lethality and cancer therapy: lessons learned from the development of PARP inhibitors. Annu Rev Med. 2015;66:455-470.

18. Wiborg O, Pedersen MS, Wind A, Berglund LE, Marcker KA, Vuust J. The human ubiquitin multigene family: some genes contain multiple directly repeated ubiquitin coding sequences. EMBO J. 1985;4(3):755-759.

19. Yau R, Rape M. The increasing complexity of the ubiquitin code. Nat Cell Biol. 2016;18(6):579-586. 
20. Cancer Genome Atlas Research Network. Integrated genomic analyses of ovarian carcinoma. Nature. 2011;474(7353):609-615.

21. Konecny GE, et al. Prognostic and therapeutic relevance of molecular subtypes in high-grade serous ovarian cancer. J Natl Cancer Inst. 2014;106(10):dju249.

22. Bibikova M, et al. High density DNA methylation array with single $\mathrm{CpG}$ site resolution. Genomics. 2011;98(4):288-295

23. Barretina J, et al. The Cancer Cell Line Encyclopedia enables predictive modelling of anticancer drug sensitivity. Nature. 2012;483(7391):603-607.

24. Cen H, Mao F, Aronchik I, Fuentes RJ, Firestone GL. DEVD-NucView488: a novel class of enzyme substrates for real-time detection of caspase-3 activity in live cells. FASEB J. 2008;22(7):2243-2252.

25. Liu X, Erikson RL. Activation of Cdc2/cyclin B and inhibition of centrosome amplification in cells depleted of Plk1 by siRNA. Proc Natl Acad Sci U S A. 2002;99(13):8672-8676.

26. Rabbitts PH, et al. Metabolism of c-myc gene products: c-myc mRNA and protein expression in the cell cycle. EMBO J. 1985;4(8):2009-2015.

27. Ciechanover A, et al. Degradation of nuclear oncoproteins by the ubiquitin system in vitro. Proc Natl Acad Sci U S A. 1991;88(1):139-143.

28. Finley D, Ozkaynak E, Varshavsky A. The yeast polyubiquitin gene is essential for resistance to high temperatures, starvation, and other stresses. Cell. 1987;48(6):1035-1046.

29. Koschwanez JH, Foster KR, Murray AW. Improved use of a public good selects for the evolution of undifferentiated multicellularity. Elife. 2013;2:e00367.

30. van Leeuwen J, et al. Exploring genetic suppression interactions on a global scale. Science. 2016;354(6312):aag0839.

31. Roig P, Gozalbo D. Depletion of polyubiquitin encoded by the UBI4 gene confers pleiotropic phenotype to Candida albicans cells. Fungal Genet Biol. 2003;39(1):70-81.

32. Nijhawan D, et al. Cancer vulnerabilities unveiled by genomic loss. Cell. 2012;150(4):842-854.

33. Solimini NL, et al. Recurrent hemizygous deletions in cancers may optimize proliferative potential. Science. 2012;337(6090):104-109.

34. Ciechanover A, Finley D, Varshavsky A. Ubiquitin dependence of selective protein degradation demonstrated in the mammalian cell cycle mutant ts85. Cell. 1984;37(1):57-66.

35. Al-Hakim A, et al. The ubiquitous role of ubiquitin in the DNA damage response. DNA Repair (Amst). 2010;9(12):1229-1240.

36. Moldovan GL, D'Andrea AD. How the fanconi anemia pathway guards the genome. Annu Rev
Genet. 2009;43:223-249.

37. Paulsen RD, et al. A genome-wide siRNA screen reveals diverse cellular processes and pathways that mediate genome stability. Mol Cell. 2009;35(2):228-239.

38. Stewart GS, et al. The RIDDLE syndrome protein mediates a ubiquitin-dependent signaling cascade at sites of DNA damage. Cell. 2009;136(3):420-434.

39. Takahashi A, Mori E, Somakos GI, Ohnishi K, Ohnishi T. Heat induces gammaH2AX foci formation in mammalian cells. Mutat Res. 2008;656(1-2):88-92.

40. Rogakou EP, Nieves-Neira W, Boon C, Pommier $\mathrm{Y}$, Bonner WM. Initiation of DNA fragmentation during apoptosis induces phosphorylation of H2AX histone at serine 139. J Biol Chem. 2000;275(13):9390-9395.

41. Ryu KY, et al. The mouse polyubiquitin gene $\mathrm{UbC}$ is essential for fetal liver development, cellcycle progression and stress tolerance. $E M B O J$. 2007;26(11):2693-2706.

42. Ryu KY, Park H, Rossi DJ, Weissman IL, Kopito RR. Perturbation of the hematopoietic system during embryonic liver development due to disruption of polyubiquitin gene $\mathrm{Ubc}$ in mice. PLoS One. 2012;7(2):e32956.

43. Sinnar SA, et al. Altered testicular gene expression patterns in mice lacking the polyubiquitin gene Ubb. Mol Reprod Dev. 2011;78(6):415-425.

44. Ryu KY, et al. The mouse polyubiquitin gene Ubb is essential for meiotic progression. Mol Cell Biol. 2008;28(3):1136-1146.

45. Lu C, Kim J, Fuller MT. The polyubiquitin gene Ubi-p63E is essential for male meiotic cell cycle progression and germ cell differentiation in Drosophila. Development. 2013;140(17):3522-3531.

46. Okazaki K, Okayama H, Niwa O. The polyubiquitin gene is essential for meiosis in fission yeast. Exp Cell Res. 2000;254(1):143-152.

47. Karnezis AN, Cho KR, Gilks CB, Pearce CL, Huntsman DG. The disparate origins of ovarian cancers: pathogenesis and prevention strategies. Nat Rev Cancer. 2017;17(1):65-74.

48. Kroeger PT, Drapkin R. Pathogenesis and heterogeneity of ovarian cancer. Curr Opin Obstet Gynecol. 2017;29(1):26-34.

49. Perets R, Drapkin R. It's totally tubular....riding the new wave of ovarian cancer research. Cancer Res. 2016;76(1):10-17.

50. Dubeau L, Drapkin R. Coming into focus: the nonovarian origins of ovarian cancer. Ann Oncol. 2013;24(suppl 8):viii28-viii35.

51. Ryu HW, Park CW, Ryu KY. Disruption of polyubiquitin gene Ubb causes dysregulation of neural stem cell differentiation with premature gliogen- esis. Sci Rep. 2014;4:7026.

52. Goldberg MS, Xing D, Ren Y, Orsulic S, Bhatia SN, Sharp PA. Nanoparticle-mediated delivery of siRNA targeting Parp1 extends survival of mice bearing tumors derived from Brca1-deficient ovarian cancer cells. Proc Natl Acad Sci US A. 2011;108(2):745-750.

53. Zuckerman JE, Davis ME. Clinical experiences with systemically administered siRNA-based therapeutics in cancer. Nat Rev Drug Discov. 2015;14(12):843-856

54. Bennett CF, Baker BF, Pham N, Swayze E, Geary RS. Pharmacology of antisense drugs. Annu Rev Pharmacol Toxicol. 2017;57:81-105.

55. McClorey G, Wood MJ. An overview of the clinical application of antisense oligonucleotides for RNA-targeting therapies. Curr Opin Pharmacol. 2015;24:52-58.

56. Helming KC, et al. ARID1B is a specific vulnerability in ARID1A-mutant cancers. Nat Med. 2014;20(3):251-254.

57. Hoffman GR, et al. Functional epigenetics approach identifies BRM/SMARCA2 as a critical synthetic lethal target in BRG1-deficient cancers. Proc Natl Acad Sci U S A. 2014;111(8):3128-3133.

58. Ogiwara $\mathrm{H}$, et al. Targeting p300 addiction in CBP-deficient cancers causes synthetic lethality by apoptotic cell death due to abrogation of MYC expression. Cancer Discov. 2016;6(4):430-445.

59. Schmittgen TD, Livak KJ. Analyzing real-time PCR data by the comparative C(T) method. Nat Protoc. 2008;3(6):1101-1108.

60. Li B, Dewey CN. RSEM: accurate transcript quantification from RNA-Seq data with or without a reference genome. BMC Bioinformatics. 2011;12:323.

61. GTEx Consortium. Human genomics. The Genotype-Tissue Expression (GTEx) pilot analysis: multitissue gene regulation in humans. Science. 2015;348(6235):648-660.

62. Fraley C, Raftery AE, Murphy TB, Scrucca L. mclust Version 4 for R: Normal Mixture Modeling for Model-Based Clustering, Classification, and Density Estimation. Technical Report Number 597. University of Washington website. https://www. stat.washington.edu/research/reports/2012/tr597. pdf. Accessed October 10, 2017.

63. Therneau T. A Package for Survival Analysis in S. version 2.38, 2015. https://CRAN.R-project.org/ package $=$ survival. Accessed October 10, 2017.

64. Sheng Q, et al. An activated ErbB3/NRG1 autocrine loop supports in vivo proliferation in ovarian cancer cells. Cancer Cell. 2010;17(3):298-310.

65. Pepe MS, Fleming TR. Weighted Kaplan-Meier statistics: a class of distance tests for censored survival data. Biometrics. 1989;45(2):497-507. 